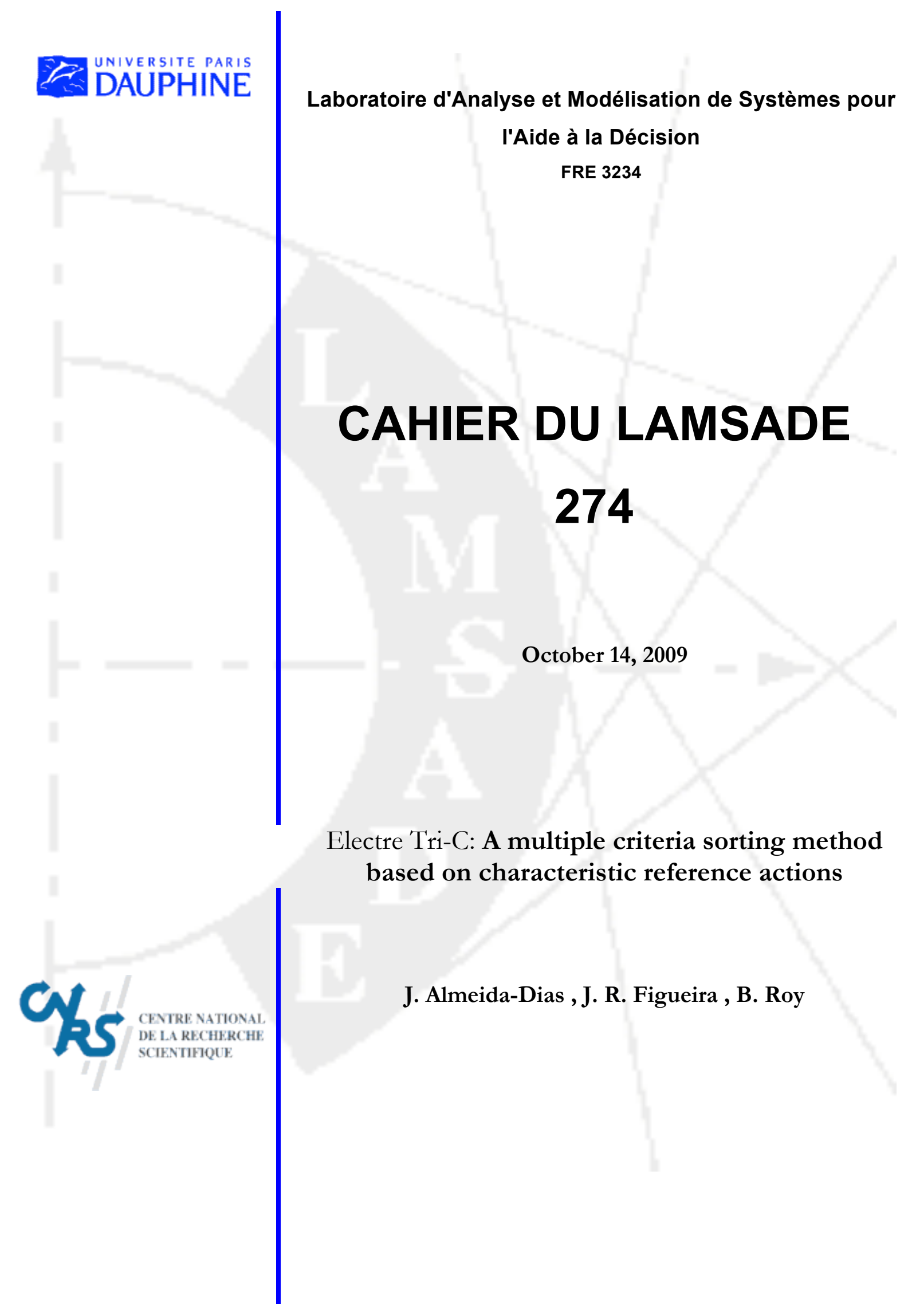




\title{
EleCtRe TRI-C: A multiple criteria sorting method based on characteristic reference actions
}

\author{
J. Almeida-Dias ${ }^{* \dagger}$, J. R. Figueira ${ }^{* \dagger}$, B. RoY ${ }^{\dagger}$
}

October 14, 2009

\footnotetext{
${ }^{*}$ CEG-IST, Instituto Superior Técnico, Technical University of Lisbon, Tagus Park, Av. Cavaco Silva, $2744-016$ Porto Salvo, Portugal. Phone: (+351)214233299. Fax: (+351)214233568. E-mails: judias@ist.utl.pt, figueira@ist.utl.pt.

${ }^{\dagger}$ LAMSADE, Université Paris-Dauphine, Place du Maréchal De Lattre de Tassigny, F-75 775 Paris Cedex 16, France. Phone: (+33)144054287. Fax: (+33)144054491. E-mail: roy@lamsade.dauphine.fr.
} 


\section{Contents}

Résumé

Abstract

1 Introduction 1

2 Concepts, definitions, and notation 2

3 Problem statement and assignment procedure 4

3.1 Additional assumptions and structural requirements . . . . . . . . . . . . . . . . . . 4

3.2 Electre Tri-C method . . . . . . . . . . . . . . . . . . . . . . 5

3.3 Foundations of Electre TRI-C . . . . . . . . . . . . . . . . . . . . . . 7

4 Properties of the ELECTRE TRI-C method 9

5 Numerical examples 11

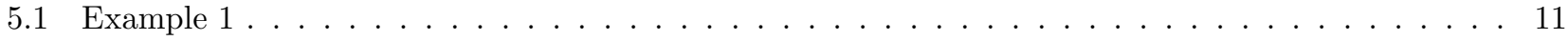

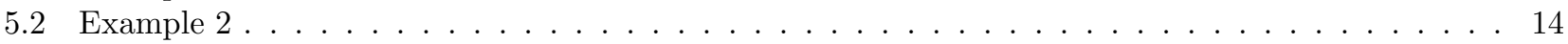

6 Electre Tri-B versus Electre Tri-C 15

7 Conclusions 16

$\begin{array}{ll}\text { Acknowledgements } & 17\end{array}$

A Appendix: Proofs 17

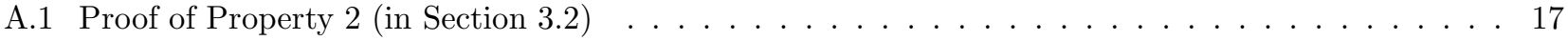

A.2 Proof of Theorem 1 (in Section 4) . . . . . . . . . . . . . . . . . . . . . . . 19

A.3 Proof of Corollary 1 (in Section 4) . . . . . . . . . . . . . . . . . . . . . . . . . 22

A.4 Proof of Theorem 2 (in Section 4) . . . . . . . . . . . . . . . . . . . . . . . . 22

A.5 Proof of Corollary 2 (in Section 4) . . . . . . . . . . . . . . . . . . . . . . . . 22

A.6 Proof of Proposition 2 (in Section 4) . . . . . . . . . . . . . . . . . . . . 22

B Appendix: Variable thresholds 23 


\title{
ELECTRE TRI-C : Une méthode multicritère de tri fondée sur des actions de référence caractéristiques
}

\begin{abstract}
Résumé
Dans cet article, une nouvelle méthode de tri, conçue selon une approche constructiviste de l'aide à la décision, est proposée. On appelle cette méthode Electre TRI-C. Comme toute méthode de tri, un ensemble de catégories doit être défini pour représenter la façon dont les actions vont être affectées à chacune des catégories, pour ensuite être traitées. Cette méthode est appropriée à des contextes d'aide à la décision où les catégories sont complètement ordonnées et chacune est définie par une seule action caractéristique de référence. L'ensemble des actions caractéristiques devrait être co-construit par un processus interactif entre l'analyste et le décideur. ELECTRE TRI-C a été conçu pour vérifier un ensemble d'exigences structurelles naturelles (la conformité, l'homogénéité, la monotonie et la stabilité), qui peuvent être considérées comme ses propriétés fondamentales. Cette méthode est constituée de deux règles, appelées la règle descendante et la règle ascendante, qui doivent être utilisées conjointement (et pas séparément). Ces règles choisissent seulement une catégorie ou un intervalle de catégories pour une possible affectation d'une action. Cette affectation dépend de la comparaison d'une telle action aux actions caractéristiques selon un niveau de crédibilité choisi. Des exemples numériques sont aussi présentés pour illustrer les résultats théoriques principaux fournis par la méthode.
\end{abstract}

Mots clé : Aide multicritère à la décision, Approche constructiviste, Problèmes de tri, EleCTRE TRI-C.

\section{EleCtRE TRI-C: A multiple criteria sorting method based on characteristic reference actions}

\begin{abstract}
In this paper, a new sorting method, following a decision aiding constructive approach, is proposed. This method is called EleCtRe TRI-C. As a sorting method, a set of categories must be defined to represent the way in which the actions that are going to be assigned to each of them should further be processed. This method is appropriate to deal with decision aiding contexts where the categories are completely ordered and each of them is defined through a single characteristic reference action. The set of characteristic actions should be co-constructed through an interactive process between the analyst and the decision maker. ELECTRE TRI-C has been conceived to verify a set of natural structural requirements (conformity, homogeneity, monotonicity, and stability), which can be viewed as its fundamental properties. This method is composed of two joint rules, called descending rule and ascending rule, which must be used conjointly (and not separately). Each one of these rules selects only one category or a range of possible categories for a possible assignment of an action. This assignment depends on the comparison of such an action to the characteristic actions according to a chosen credibility level. Numerical examples are also presented in order to illustrate the main theoretical results provided by the method.
\end{abstract}

Keywords: Multiple Criteria Decision Aiding, Constructive Approach, Sorting, Electre TrI-C, Decision Support. 


\section{Introduction}

Different decision problems require different approaches to solve them. We are interested in decision aiding contexts in which the objects of a decision (actions, alternatives, ...) must be sorted, or assigned to a set of categories. Let us notice that the term "decision aiding" is used instead of "decision support", "decision making", or "decision analysis" to avoid any simplistic assimilation. The assignment of the actions to the categories is based on the evaluation of each action according to multiple criteria. In such decision aiding sorting contexts, several assumptions are appropriate to the manner that the decision aiding is considered:

Assumption 1 The set of categories to which the actions must be assigned to is completely ordered (from the best to the worst, from the highest priority to the lowest priority, from the most risky to the least risky, from the most consensual to the least consensual, and so on).

Assumption 2 Each category is defined a priori to receive actions, which will be or might be processed in the same way (at least in a first step).

Assumption 3 Each category is defined by a reference action, which is the most representative one, called characteristic reference action, or characteristic action.

Let us suppose that the decision maker is able, in interaction along with the analyst, to provide, for each criterion, the performance of each characteristic action. Let us notice that the term "decision maker" represents those in whose name or for whom the decision aiding must be given and the "analyst" represents a facilitator of the decision aiding process, which must perform her/his role in interaction with the decision maker.

For instance, the actions can be patients waiting for an Assisted Reproductive Technology (ART) treatment, credit demand files, risk zones, candidats for a job, environmental mesures, or R\&D projects. In case of ART treatments, the categories can be defined as the number of embryos to be transferred to the uterus of a women in order to achieve a pregnancy and to reduce the risk of multiple pregnancies at the same time. In the case of credit demand files, such actions can be accepted without additional information, accepted subject to additional information, sent to a particular department for further analysis, rejected under certain conditions, or rejected with no additional conditions at all. The method proposed in this paper, designated ElECTRE TRI-C, seems appropriate to deal with this kind of situations in which the objective is not intend to discover the pre-existing categories where the studied actions would naturally be assigned to, but to help decision makers to assign each action to a certain category. This category should be the most appropriate taking into account its characteristics and the definition of the set of categories.

ELECTRE TRI-C was designed to be used within the framework of a constructive approach (see Roy 1993). It means that the decision aiding assignment model is, in a certain sense, at least co-constructed through an interactive process between the analyst and the decision maker. Firstly, this co-construction process is related to the definition of the characteristic actions, which represents the set of categories that the decision maker considers necessary to use for regrouping the actions. Secondly, this co-construction process also concerns the way of defining criteria, by assigning values to the indifference and preference thresholds, the veto thresholds (if they exist), and an intrinsic weight to each criterion in the pre-defined coherent family of criteria. Finally, as shown in the next sections, it is necessary that the analyst in agreement with the decision maker chooses a minimum credibility level to validate or not a comparison statement.

ElECTRE TRI-C is composed of two joint rules, called descending rule and ascending rule. Each one of these rules selects only one category for a possible assignment of an action. They are used conjointly in order to highlight the highest category and the lowest category, which can appear potentially appropriate to receive an action. These two extreme categories can be the same. When they differ, this means that the assignment of such an action remains ill-determined within a range of possible categories taking into account the way that the set of characteristic actions defines the categories.

Since the late seventies, several procedures have been proposed for sorting problems (according to the Assumption 1) as the following ones: trichotomic segmentation (Moscarola and Roy 1977), UtADIS (Devaud et al. 1980; Zopounidis and Doumpos 2002), N-Tomic (Massaglia and Ostanello 1991), Electre Tri (Yu 1992; Roy and Bouyssou 1993), filtering by preference (denoted here FPP) (Perny 1998), multi-profile sorting by intersection sets (Norese and Viale 2002), PAIRClas (Doumpos and Zopounidis 2004), and SMAA-TRI (Tervonen et al. 2009). Let us notice that the key concepts which are mainly related, for instance, to the FPP procedure (Perny 1998), the ProAfTn method (Belacel 2000), and the sorting by preference closeness method (denoted here CLOSORT) 
(Fernandez et al. 2008) were firstly proposed by Słowiński and Stefanowski (1994) in order to build a set of decision rules based on both multiple criteria and multiple attributes according to the rough set theory framework.

The Electre TRI method can be applied to decision aiding contexts in which the Assumptions 1 and 2 are fulfilled, while replacing the Assumption 3 by the following one:

Assumption 4 Each category is defined by two reference actions, which represent its lower and upper bounds, called boundary reference actions, or boundary actions.

The boundary actions are introduced for modeling the frontiers between two consecutive categories. This means that the lower boundary action of a better category is also the upper boundary action of the worse consecutive category (categories are closed from below). The use of ElECTRE TRI suppose that the decision maker is able, in interaction along with the analyst, to provide, for each criterion, the performance of each boundary action.

ELECTRE TRI-C is, therefore, a new sorting method: the actions to be assigned are not compared to reference actions that represent lower and upper bounds of the categories, but instead they are compared to reference actions that contain the representative characteristics of each category. Each one of such categories must be defined in EleCtRe TRI-C by a single characteristic action which defines it, instead of a pair of boundary actions like in Electre Tri. To avoid some misunderstanding, the EleCtre TrI method based on boundary actions will be designated henceforth by ELECTRE TRI-B.

The rest of this paper is organized as follows. Section 2 introduces and reviews some concepts, their definitions, and notation. Section 3 is devoted to the proposed ElECTRE TRI-C method, which contains the additional assumptions, the natural structural requirements, and the two joint rules and their foundations. Section 4 presents the properties of ElECTRE TRI-C, including the analysis of the assignment results. Section 5 provides two numerical examples in order to illustrate the theoretical results presented in this paper. Section 6 presents a comparison with ELECTRE TRI-B according to the basic assumptions, the related assignment rules, and the role of the reference actions, which are used to define the categories. Finally, the last section offers our concluding remarks and some avenues for future research.

\section{Concepts, definitions, and notation}

Let $\left\{a_{1}, a_{2}, \ldots, a_{i}, \ldots\right\}$ denote the potential actions. This set of actions, denoted $A$, can be completely known $a$ priori or it may appear progressively during the decision aiding process. The objective is to assign these actions to a set of completely ordered categories, denoted $\left\{C_{1}, \ldots, C_{h}, \ldots, C_{q}\right\}$, with $q \geq 2$. Suppose that a coherent family of $n$ criteria, denoted $F=\left\{g_{1}, \ldots, g_{j}, \ldots, g_{n}\right\}$, with $n \geq 3$, has been defined in order to evaluate any action considered to be assigned to a certain category (see Roy 1996). In what follows, assume, without loss of generality, that all criteria $g_{j} \in F$ are to be maximized, which means that the preference increases when the criterion performance increases too.

Let us consider also that each criterion $g_{j}$ will be considered as a pseudo-criterion, which means that two thresholds are associated to $g_{j}$ : an indifference threshold, $q_{j}$, and a preference threshold, $p_{j}$, such that $p_{j} \geq q_{j} \geq 0$. These thresholds are introduced in order to take into account the imperfect character of the data from the computation of the performances $g_{j}(a)$, for all $a \in A$, as well as the arbitrariness that affects the definition of the criteria. Based on the definition of such thresholds, the following binary relations can be derived for each criterion:

i) $\left|g_{j}(a)-g_{j}\left(a^{\prime}\right)\right| \leq q_{j}$ represents a non-significant advantage of one of the two actions over the other, meaning that $a$ is indifferent to $a^{\prime}$ according to $g_{j}$, denoted $a I_{j} a^{\prime}$. Let $C\left(a I a^{\prime}\right)$ be the subset of criteria such that $a I_{j} a^{\prime}$.

ii) $g_{j}(a)-g_{j}\left(a^{\prime}\right)>p_{j}$ represents a significant advantage of $a$ over $a^{\prime}$, meaning that $a$ is strictly preferred to $a^{\prime}$ according to $g_{j}$, denoted $a P_{j} a^{\prime}$. Let $C\left(a P a^{\prime}\right)$ be the subset of criteria such that $a P_{j} a^{\prime}$.

iii) $q_{j}<g_{j}(a)-g_{j}\left(a^{\prime}\right) \leq p_{j}$ represents an ambiguity zone. The advantage of $a$ over $a^{\prime}$ is a little large to conclude about an indifference between $a$ and $a^{\prime}$, but this advantage is not enough to conclude about a strict preference in favour of $a$. This means that there is an hesitation between indifference and strict preference. In such a case, $a$ is weakly preferred to $a^{\prime}$, denoted $a Q_{j} a^{\prime}$. Let $C\left(a Q a^{\prime}\right)$ be the subset of criteria such that $a Q_{j} a^{\prime}$. 
Let us notice that $q_{j}$ can be null and/or equal to $p_{j}$. Furthermore, if $p_{j}=0$, then any difference of performances in favor of one of the two actions over the other can be considered as significant for a strict preference on criterion $g_{j}$. However, this is not always true due to the imperfect character of the data and the arbitrariness that affects the definition of the criteria when using a continuous scale or even a discrete one for criterion $g_{j} \in F$. In the latter case, the difference between two discrete consecutive possible performances can, for instance, be considered as a weak preference and even as a non-significant difference. If such a difference is associated to strict preference, then it can be used to define $p_{j}$. In what follows, the possibility of $p_{j}=0$ is not excluded, but such a case must be considered as unusually realistic.

When using the outranking concept, the main idea is that " $a$ outranks $a^{\prime}$ " according to the criterion $g_{j}$, denoted $a S_{j} a^{\prime}$, if " $a$ is at least as good as $a^{\prime \prime}$ " on criterion $g_{j}$. This is validated, without ambiguity, when $g_{j}(a)-g_{j}\left(a^{\prime}\right) \geq-q_{j}$. But, when $-p_{j} \leq g_{j}(a)-g_{j}\left(a^{\prime}\right)<-q_{j}$, the possibility of indifference between $a$ and $a^{\prime}$ cannot be excluded. This indifference is less and less credible when $g_{j}(a)-g_{j}\left(a^{\prime}\right)$ moves closer to $-p_{j}$.

A single vector of weights, denoted $w_{j}$, such that $w_{j}>0, j=1, \ldots, n$, is associated to the set of criteria. Assume that $\sum_{j=1}^{n} w_{j}=1$. Thus, the comprehensive concordance index, denoted $c\left(a, a^{\prime}\right)$, is defined as follows:

$$
c\left(a, a^{\prime}\right)=\sum_{j \in C\left(a P a^{\prime}\right)} w_{j}+\sum_{j \in C\left(a Q a^{\prime}\right)} w_{j}+\sum_{j \in C\left(a I a^{\prime}\right)} w_{j}+\sum_{j \in C\left(a^{\prime} Q a\right)} w_{j} \varphi_{j},
$$

where

$$
\varphi_{j}=\frac{g_{j}(a)-g_{j}\left(a^{\prime}\right)+p_{j}}{p_{j}-q_{j}} \in[0,1[.
$$

Let us notice that in the family of ELECTRE methods, the intrinsic weights $w_{j}$ are interpreted as a power of vote. $\varphi_{j}$ represents the way in which such a power of vote decrease according to the criteria $g_{j} \in C\left(a^{\prime} Q a\right)$.

A vector of veto thresholds, denoted $v_{j}$, such that $v_{j} \geq p_{j}$ can also be associated to the set of criteria. Thus, the partial discordance indices, denoted $d_{j}\left(a, a^{\prime}\right), j=1, \ldots, n$, are defined as follows:

$$
d_{j}\left(a, a^{\prime}\right)= \begin{cases}1 & \text { if } g_{j}(a)-g_{j}\left(a^{\prime}\right)<-v_{j}, \\ \frac{g_{j}(a)-g_{j}\left(a^{\prime}\right)+p_{j}}{p_{j}-v_{j}} & \text { if }-v_{j} \leq g_{j}(a)-g_{j}\left(a^{\prime}\right)<-p_{j} \\ 0 & \text { if } g_{j}(a)-g_{j}\left(a^{\prime}\right) \geq-p_{j} .\end{cases}
$$

Finally, let $\sigma\left(a, a^{\prime}\right)$ denote the credibility of the comprehensive outranking of $a$ over $a^{\prime}$, which reflects the strength of the statement " $a$ outranks $a^{\prime}$ " (denoted $a S a^{\prime}$ ) when taking all the criteria from $F$ into account. The credibility index is defined as follows:

$$
\sigma\left(a, a^{\prime}\right)=c\left(a, a^{\prime}\right) \prod_{j=1}^{n} T_{j}\left(a, a^{\prime}\right)
$$

where

$$
T_{j}\left(a, a^{\prime}\right)= \begin{cases}\frac{1-d_{j}\left(a, a^{\prime}\right)}{1-c\left(a, a^{\prime}\right)} & \text { if } d_{j}\left(a, a^{\prime}\right)>c\left(a, a^{\prime}\right), \\ 1 & \text { otherwise. }\end{cases}
$$

The indifference, preference, and veto thresholds have been presented as constants in order to simplify the presentation of the EleCtRE TRI-C method. However, in practice, these thresholds can vary according to the performances $g_{j}(a)$ or $g_{j}\left(a^{\prime}\right)$. The way to generalize the Formulas (2.1)-(2.3) for taking into account variable thresholds, which is often absolutely required in several real-world problems, is analyzed in the Appendix B (for more details, see Roy and Vincke 1984, Roy 1996, p. 184-94, Roy 1991, Yu 1992, and Roy and Bouyssou 1993).

Let $\lambda$ denote a credibility level as the minimum degree of credibility, $\sigma\left(a, a^{\prime}\right)$, which is considered or judged necessary by the decision maker to validate or not the statement " $a$ outranks $a$ " taking all the criteria from $F$ into account (Definition 1). In general, this minimum credibility level takes a value within the range $[0.5,1]$.

\section{Definition 1 ( $\lambda$-binary relations)}

a) $\lambda$-outranking: $a S^{\lambda} a^{\prime} \Leftrightarrow \sigma\left(a, a^{\prime}\right) \geq \lambda$.

b) $\lambda$-preference: $a P^{\lambda} a^{\prime} \Leftrightarrow \sigma\left(a, a^{\prime}\right) \geq \lambda \wedge \sigma\left(a^{\prime}, a\right)<\lambda$. 
c) $\lambda$-indifference: $a I^{\lambda} a^{\prime} \Leftrightarrow \sigma\left(a, a^{\prime}\right) \geq \lambda \wedge \sigma\left(a^{\prime}, a\right) \geq \lambda$.

d) $\lambda$-incomparability: $a R^{\lambda} a^{\prime} \Leftrightarrow \sigma\left(a, a^{\prime}\right)<\lambda \wedge \sigma\left(a^{\prime}, a\right)<\lambda$.

Let us notice that the $\lambda$-indifference is obtained as soon as the statements " $a$ outranks $a^{\prime \prime}$ " and " $a$ ' outranks $a "$ are both validated. Thus, $\lambda$-indifference does not mean that $a$ and $a^{\prime}$ are indifferent on all the criteria. This mean that $a$ is $\lambda$-indifferent to $a^{\prime}$ if $a$ and $a^{\prime}$ have almost the same performances on at least a sufficient subset of criteria in order to achieve the chosen credibility level, $\lambda$. Similarly, the $\lambda$-incomparability is obtained when both the statements " $a$ outranks $a^{\prime \prime}$ " and " $a$ ' outranks $a$ " are not validated. This mean that $a$ is $\lambda$-incomparable to $a^{\prime}$ if for a subset of criteria $a$ has significantly better performances than those of $a^{\prime}$, while for the remaining criteria the performances of $a^{\prime}$ are significantly better than those of $a$, where no one of these two subsets of criteria achieve the chosen credibility level, $\lambda$.

\section{Problem statement and assignment procedure}

The aim of this section is to present the ElECTRE TRI-C method, including additional assumptions, structural requirements, two joint rules as well as their foundations.

\subsection{Additional assumptions and structural requirements}

Let $b_{h}$ denote a characteristic action introduced to define category $C_{h}$. Assume that the reference actions $b_{h}$, $h=1, \ldots, q$, have been defined through a co-construction process (see Section 1). Notice that $C_{1}$ is the worst category and $C_{q}$ is the best one, with $q \geq 2$. Let $B=\left\{b_{0}, b_{1}, \ldots, b_{h}, \ldots, b_{q}, b_{q+1}\right\}$ denote the set of $(q+2)$ characteristic actions, where $b_{0}$ and $b_{q+1}$ are two particular characteristic actions defined as follows: $g_{j}\left(b_{0}\right)$ is the worst possible performance on criterion $g_{j}$, and $g_{j}\left(b_{q+1}\right)$ is the best possible performance on the same criterion $g_{j}$, for all $g_{j} \in F$. The worst and the best possible performances must be chosen such that, for any action $a$, one has $g_{j}\left(b_{0}\right)<g_{j}(a)<g_{j}\left(b_{q+1}\right)$, for all $g_{j} \in F$. Moreover, for all $g_{j} \in F$, one has $g_{j}\left(b_{1}\right)-g_{j}\left(b_{0}\right)>0$ and $g_{j}\left(b_{q+1}\right)-g_{j}\left(b_{q}\right)>0$.

According to the ordered character of the categories, by definition of the characteristic actions, $b_{h+1}$ must be distinct from $b_{h}$ and $b_{h+1}$ strictly dominates $b_{h}, h=1, \ldots,(q-1)$. Let us recall that this (strict) dominance condition is defined as follows:

$$
\forall j, g_{j}\left(b_{h+1}\right)-g_{j}\left(b_{h}\right) \geq 0 \text { and } \exists j, g_{j}\left(b_{h+1}\right)-g_{j}\left(b_{h}\right)>0, h=1, \ldots,(q-1) .
$$

When considering the possible minimum differences in the performances of the characteristic actions, the (strict) dominance condition is not enough to distinguish two consecutive categories. If for all the criteria from $F$ such that $0 \leq g_{j}\left(b_{h+1}\right)-g_{j}\left(b_{h}\right) \leq q_{j}$, then the characteristic actions $b_{h+1}$ and $b_{h}$ are not adequate to define two consecutive categories clearly distinct. In such a case, $\sigma\left(b_{h}, b_{h+1}\right)=1$.

Two characteristic actions, $b_{h+1}$ and $b_{h}$, define two distinct categories only if at least $b_{h+1}$ is weakly preferred to $b_{h}$ according to at least one criterion. This implies that $\sigma\left(b_{h}, b_{h+1}\right)<1$. Therefore, the set of characteristic actions, $B$, must fulfill the weak separability (Condition 1). If such a condition is not fulfilled, the analyst must improve the interaction process with the decision maker in order to obtain a consistent set $B$.

Condition 1 (Weak separability) The set of characteristic actions, B, fulfills the weak separability condition if and only if $\sigma\left(b_{h}, b_{h+1}\right)<1, h=1, \ldots,(q-1)$.

According to some practical situations, this condition can be judged, by the decision maker, too weak for defining significant distinct categories through the characteristic actions. In such a case, it is often desirable to impose a strong condition to the set $B$, defined as follows:

Condition 2 (Strict separability) The set of characteristic actions, B, fulfills the strict separability condition if and only if $\sigma\left(b_{h}, b_{h+1}\right)<\frac{1}{2}, h=1, \ldots,(q-1)$.

However, in certain cases, the set $B$ can also fulfill a stronger condition than the above two separability conditions, which is defined as follows:

Condition 3 (Hyper-strict separability) The set of characteristic actions, B, fulfills the hyper-strict separability condition if and only if $\sigma\left(b_{h}, b_{h+1}\right)=0, h=1, \ldots,(q-1)$. 
This last condition is equivalent to impose that: if $p_{j}>0$, then $g_{j}\left(b_{h+1}\right)-g_{j}\left(b_{h}\right) \geq p_{j}$; otherwise, $g_{j}\left(b_{h+1}\right)-$ $g_{j}\left(b_{h}\right)>0, j=1, \ldots, n ; h=1, \ldots,(q-1)$. Let us notice that a similar condition to the hyper-strict separability, called distinguishability condition, is used in Perny (1998, p. 154).

The following structural requirements (Definition 2) can be viewed as the desirable properties, which are imposed a priori to the ELECTRE TRI-C method.

\section{Definition 2 (Structural requirements)}

a) Conformity: each characteristic action $b_{h}$ must be assigned to category $C_{h}, h=1, \ldots, q$.

b) Homogeneity: two actions must be assigned to the same category when they have the same outranking credibility indices with respect to the characteristic actions.

c) Monotonicity: if an action a strictly dominates $a^{\prime}$, then a is assigned at least to the same category $a^{\prime}$ is assigned to.

d) Stability: when applying either a merging or a splitting operation (see Definition 3), the actions previously assigned to the non-modified categories will be assigned to the same categories or, possibly, to the new categories, after modification. More precisely:

1) After merging two consecutive categories:

- any action previously assigned to a non-adjacent category to the modified ones will remain in the same category;

- any action previously assigned to an adjacent category to the modified ones will either be assigned to the same category or to the new category;

- any action previously assigned to a merged category will either be assigned to the new category or to an adjacent category.

2) After splitting a category into two new consecutive categories:

- any action previously assigned to a non-adjacent category to the modified one will remain in the same category;

- any action previously assigned to an adjacent category to the modified one will either be assigned to the same category or to a new category;

- any action previously assigned to the split category will either be assigned to a new category or to an adjacent category.

\section{Definition 3 (Merging and splitting operations)}

a) Merging operation: two consecutive categories, $C_{h}$ and $C_{h+1}$, will be merged to become a new one, $C_{h}^{\prime}$, while introducing a new characteristic action, $b_{h}^{\prime}$, such that $g_{j}\left(b_{h}^{\prime}\right)-g_{j}\left(b_{h}\right) \geq 0$ and $g_{j}\left(b_{h+1}\right)-g_{j}\left(b_{h}^{\prime}\right) \geq 0$, for all $g_{j} \in F$.

b) Splitting operation: the category $C_{h}$ will be split into two new consecutive categories, $C_{h}^{\prime}$ and $C_{h}^{\prime \prime}$, while introducing two new characteristic actions, $b_{h}^{\prime}$ and $b_{h}^{\prime \prime}$, respectively, such that $\sigma\left(b_{h}^{\prime \prime}, b_{h+1}\right)<1, \sigma\left(b_{h}^{\prime}, b_{h}^{\prime \prime}\right)<1$, $\sigma\left(b_{h-1}, b_{h}^{\prime}\right)<1, g_{j}\left(b_{h}^{\prime \prime}\right)-g_{j}\left(b_{h}\right) \geq 0$, and $g_{j}\left(b_{h}\right)-g_{j}\left(b_{h}^{\prime}\right) \geq 0$, for all $g_{j} \in F$.

It should be noticed that adding or removing a category are particular cases of these two basic operations.

\subsection{Electre Tri-C method}

This section presents the two proposed joint rules of the ELECTRE TRI-C method, which must be used conjointly (and not separately). When using a selecting function, denoted $\rho(a, b)$, subjected to some properties (see below), these two rules are defined as follows:

Definition 4 (Descending rule) Choose a credibility level, $\lambda\left(\frac{1}{2} \leq \lambda \leq 1\right)$. Decrease $h$ from $(q+1)$ until the first value, $t$, such that $\sigma\left(a, b_{t}\right) \geq \lambda$ :

a) For $t=q$, select $C_{q}$ as a possible category to assign action a. 
b) For $0<t<q$, if $\rho\left(a, b_{t}\right)>\rho\left(a, b_{t+1}\right)$, then select $C_{t}$ as a possible category to assign a; otherwise, select $C_{t+1}$.

c) For $t=0$, select $C_{1}$ as a possible category to assign a.

In the descending rule, a category is selected taking into account that: $b_{t}$ is the highest characteristic action such that the statement " $a$ outranks $b_{t}$ " is validated with the chosen credibility level, $\lambda$. In such a case, the possibility of the assignment of action $a$ to the pre-selected category $C_{t}$ must be examined. Nevertheless, taking into account the manner that the characteristic actions $b_{t}$ and $b_{t+1}$ were defined, the assignment of action $a$ to $C_{t+1}$ is an alternative that must also be examined (in such a case, the statement " $a$ outranks $b_{t+1}$ " is not validated with the chosen credibility level, $\lambda$ ) because $b_{t+1}$ was not defined to play the role of an upper bound for category $C_{t}$.

Definition 5 (Ascending rule) Choose a credibility level, $\lambda\left(\frac{1}{2} \leq \lambda \leq 1\right)$. Increase $h$ from zero until the first value, $k$, such that $\sigma\left(b_{k}, a\right) \geq \lambda$ :

a) For $k=1$, select $C_{1}$ as a possible category to assign action a.

b) For $1<k<(q+1)$, if $\rho\left(a, b_{k}\right)>\rho\left(a, b_{k-1}\right)$, then select $C_{k}$ as a possible category to assign a; otherwise, select $C_{k-1}$.

c) For $k=(q+1)$, select $C_{q}$ as a possible category to assign a.

In the ascending rule, a category is selected taking into account that: $b_{k}$ is the lowest characteristic action such that the statement " $b_{k}$ outranks $a$ " is validated with the chosen credibility level, $\lambda$. In such a case, the possibility of the assignment of action $a$ to the pre-selected category $C_{k}$ must be examined. Nevertheless, taking into account the manner that the characteristic actions $b_{k}$ and $b_{k-1}$ were defined, the assignment of action $a$ to $C_{k-1}$ is an alternative that must also be examined (in such a case, the statement " $b_{k-1}$ outranks $a$ " is not validated with the chosen credibility level, $\lambda$ ) because $b_{k-1}$ was not defined to play the role of a lower bound for category $C_{k}$.

Therefore, each one of the two joint rules requires the selecting function, $\rho(a, b)$, which allows to choose between the two consecutive categories where an action $a$ can be assigned to. Due to the role played by this selecting function in the two joint rules, it must fulfill the two following properties:

\section{Property 1}

a) $\rho(a, b)$ is a function of $\sigma(a, b)$ and $\sigma(b, a)$, where $b$ is a characteristic reference action.

b) Let $C_{h}$ be the pre-selected category for a possible assignment of action a. The selection of $C_{h}$ (instead of an adjacent category, which is also candidate) is justified if and only if $\rho\left(a, b_{h}\right)$ is strictly greater than $\rho(a, b)$ for the adjacent category. Consequently, if a is the same as $b_{h}$, then $\rho\left(b_{h}, b_{h}\right)$ must be the best of the two values (the equality being excluded).

Property 2 Let $a$ and $a^{\prime}$ be two actions that allow to pre-select the same category. If a strictly dominates $a^{\prime}$, then $\rho\left(a, b_{h}\right)>\rho\left(a, b_{h+1}\right) \Rightarrow \rho\left(a^{\prime}, b_{h}\right)>\rho\left(a^{\prime}, b_{h+1}\right)$. This implication is equivalent, by logic negation, to $\rho\left(a^{\prime}, b_{h+1}\right) \geq \rho\left(a^{\prime}, b_{h}\right) \Rightarrow \rho\left(a, b_{h+1}\right) \geq \rho\left(a, b_{h}\right)$.

Property 1 is evident since a) stand from the conditions in which $\rho(a, b)$ is founded, and $b$ ) is necessary so that the selected categories by each one of the two joint rules have a meaning in the ELECTRE TRI-C assignment procedure. Property 2 is necessary in order to fulfill the monotonicity with respect to each one of the two joint rules.

The EleCtRe TRI-C assignment procedure allows to select two possible categories to which an action $a$ can be assigned. Such an action $a$ can effectively be assigned, by the decision maker, to:

- a single category, when the two selected categories are the same;

- one of the two selected categories, when such categories are consecutive;

- one of the two selected categories or one of the intermediate categories, when such categories are not consecutive. 


\subsection{Foundations of EleCTRE TRI-C}

This section provides the foundations for the ELECTRE TRI-C assignment procedure based on the answers of four key questions:

1) Why to found an assignment procedure on the basis of the outranking credibility indices of types $\sigma\left(a, b_{h}\right)$ and $\sigma\left(b_{h}, a\right)$ ?

The sorting problem analyzed in this paper introduces three fundamental features, or aspects:

i) the categories, in which the actions must be assigned to, are ordered;

ii) to operate on this assignment, the performances of such actions are evaluated according to several criteria;

iii) the actions to be assigned are compared with the characteristic actions, which define the set of categories. There is, therefore, a comparison with norms and not between pairs of actions to be assigned to such categories.

The assignment of an action $a$ to a category $C_{h}$ must naturally be based on the manner that such an action $a$ compares itself with the characteristic actions $b_{h}$, which is used to define the category $C_{h}$. In order to properly take into account the three fundamental features of the problem, an assignment procedure could be founded on the "more or less high credibility" of the following statements: "an action $a$ outranks a characteristic action $b_{h}$ " and "a characteristic action $b_{h}$ outranks an action $a$ ". The credibility indices $\sigma\left(a, b_{h}\right)$ and $\sigma\left(b_{h}, a\right)$ are appropriate to model this "more or less high credibility". Taking into account the manner that the characteristic action $b_{h}$ is defined, the category $C_{h}$ can receive actions $a$ that outrank $b_{h}$ as well as actions $a$ that are outranked by $b_{h}$.

2) Why two joint assignment rules for giving a range of possible consecutive categories in which an action a can be assigned to?

Taking the chosen credibility level, $\lambda$, into account, it can exist some actions $a$ such that one of the two following situations occurs (see Proposition 1, in Section 4):

i) there is at least one characteristic action $b_{h}$, which is neither outranked by action $a$ nor outranks $a$ (i.e., $a$ and $b_{h}$ are $\lambda$-incomparable);

ii) there are more than one characteristic action $b_{h}$, which are outranked by action $a$ and outrank $a$ at the same time (i.e., $a$ and $b_{h}$ are $\lambda$-indifferent).

In the two above situations, it seems to us inappropriate to assign such an action $a$ to only one category. But, on the contrary, it seems appropriate to search for the lowest category and the highest category likely to receive the action $a$, including all the possible intermediate categories (if they exist). Taking into account the way that the categories are defined, it can also exist some situations, which differ from those described above (see i) and ii)), so that several possibilities of an assignment can be derived. Instead of choosing one of the several categories, based on a more or less arbitrary assignment rule, we think that this choice must be done by the decision maker. In such a case, a category must be chosen by the decision maker according to the performances of the action $a$, her/his experience, and the set of the next processing operations, which is associated to the definition of the selected categories.

3) Why to use both descending and ascending rules conjointly?

\section{Definition 6 (Transposition operation)}

Let us denote $p$ the problem introduced in Section 3.1. Consider a new problem, denoted $p^{\prime}$, verifying:

a) The set of new criteria is $F^{\prime}=\left\{g_{j}^{\prime}, j=1, \ldots, n\right\}$, such that each new criterion, $g_{j}^{\prime}$, is obtained from the problem $p$ by the inversion of the preference direction of the criterion $g_{j} \in F$.

b) The set of new categories is $C^{\prime}=\left\{C_{h}^{\prime}, h=1, \ldots, q\right\}$, such that $C_{h}^{\prime}=C_{q+1-h}, h=1, \ldots, q$. In this case, the worst category of the problem $p, C_{1}$, becomes the best one of the problem $p^{\prime}, C_{q}^{\prime}$, and the best category of the problem $p, C_{q}$, becomes the worst one of the problem $p^{\prime}, C_{1}^{\prime}$.

c) The performances of all the potential actions and all the characteristic actions (and notation) remain the same as in the problem $p$. 
Problem $p^{\prime}$ (transposed from problem $p$ ) is equivalent to $p$. Let $\sigma^{\prime}\left(a, b_{h}\right)$ and $\sigma^{\prime}\left(b_{h}, a\right)$ denote the new outranking credibility indices obtained for the equivalent problem $p^{\prime}$. It is trivial to prove that, for all actions $a$ and $b_{h}$, one has: $\sigma^{\prime}\left(a, b_{h}\right)=\sigma\left(b_{h}, a\right)$ and $\sigma^{\prime}\left(b_{h}, a\right)=\sigma\left(a, b_{h}\right)$. When the descending rule (Definition 4) is applied to the problem $p^{\prime}$, the credibility indices $\sigma^{\prime}\left(a, b_{h}\right)$ and $\sigma^{\prime}\left(b_{h}, a\right)$ are used to play the same role as the credibility indices $\sigma\left(b_{h}, a\right)$ and $\sigma\left(a, b_{h}\right)$ in the ascending rule (Definition 5) applied to the problem $p$.

Therefore, the transposition operation shows a way to replace the descending rule by the ascending rule. There is no reason to choose only one of the two proposed joint rules, since they are not significantly distinct. On the contrary, when the two joint rules are applied conjointly, they either clearly show a single category where an action $a$ can be assigned to if there is no ambiguity in such an assignment or the lowest category and the highest category likely to receive an action $a$, while such an assignment remain ill-determined within such a range.

4) What kind of selecting function, $\rho(a, b)$, can preserve the role of the characteristic actions $b$ ?

The selecting function, $\rho(a, b)$, required in the joint rules of ELECTRE TRI-C can specifically be defined in several ways. Therefore, Properties 1 and 2 do not determine a unique shape for the selecting function, $\rho(a, b)$. We propose to study the following one (see also Figure 1):

$$
\rho(a, b)=\min \{\sigma(a, b), \sigma(b, a)\} .
$$

Let us prove that the min selecting function (3.2) fulfills the two necessary properties as stated above (see Section 3.2). Regarding Property 1, we will considered successively each one of the two joint rules.

i) The selection is performed by the descending rule (Definition 4):

$C_{t}$ is pre-selected if and only if the statement " $a$ outranks $b_{t+1}$ " is not validated with the chosen credibility level, $\lambda$, while the statement " $a$ outranks $b_{t}$ " is validated with the same chosen credibility level, $\lambda$. In other words, if and only if

$$
\sigma\left(a, b_{t+1}\right)<\lambda \leq \sigma\left(a, b_{t}\right) .
$$

Arguments in favor of the selection of $C_{t}$ (instead of $\left.C_{t+1}\right)$ : this selection seems to be more and more justified when the credibility of the statement " $b_{t}$ outranks $a$ " moves closer to 1 . In other words, when $\sigma\left(b_{t}, a\right)$ becomes higher.

Arguments against the selection of $C_{t}$ (instead of $C_{t+1}$ ): this selection seems to be less and less justified when the credibility of the statement " $a$ outranks $b_{t+1}$ " moves closer to $\lambda$. In other words, when $\sigma\left(a, b_{t+1}\right)$ becomes higher. According to such arguments, when using the descending rule:

- the selection of $C_{t}$ is justified if and only if $\sigma\left(b_{t}, a\right)>\sigma\left(a, b_{t+1}\right)$;

- on the contrary, if $\sigma\left(a, b_{t+1}\right) \geq \sigma\left(b_{t}, a\right)$, then the selection of $C_{t+1}$ is justified.

The min selecting function (3.2) leads precisely to the same selection as analyzed above for the descending rule for the following reasons:

- according to $a$ ) of Corollary 3 (see Appendix A.1), $C_{t}$ is selected if and only if $\sigma\left(b_{t}, a\right)>\sigma\left(a, b_{t+1}\right)$ (the inequality $\sigma\left(a, b_{t+1}\right)<\sigma\left(a, b_{t}\right)$ being always verified, see the inequalities (3.3) above).

- according to $b$ ) of Lemma 1 (see Appendix A.1), $C_{t+1}$ is selected if and only if $\sigma\left(a, b_{t+1}\right) \geq \sigma\left(b_{t}, a\right)$ (the equality $\sigma\left(a, b_{t+1}\right)=\sigma\left(a, b_{t}\right)$ being excluded here, see the inequalities (3.3) above).

ii) The selection is performed by the ascending rule (Definition 5):

Let $C_{k+1}$ be the pre-selected category. This category is pre-selected if and only if the statement " $b_{k}$ outranks $a$ " is not validated with the chosen credibility level, $\lambda$, while the statement " $b_{k+1}$ outranks $a$ " is validated with the same chosen credibility level, $\lambda$. In other words, if and only if

$$
\sigma\left(b_{k}, a\right)<\lambda \leq \sigma\left(b_{k+1}, a\right) .
$$

Arguments in favor of the selection of $C_{k+1}$ (instead of $C_{k}$ ): this selection seems to be more and more justified when the credibility of the statement " $a$ outranks $b_{k+1}$ " moves closer to 1 . In other words, when $\sigma\left(a, b_{k+1}\right)$ becomes higher.

Arguments against the selection of $C_{k+1}$ (instead of $C_{k}$ ): this selection seems to be less and less justified when the credibility of the statement " $b_{k}$ outranks $a$ " moves away from $\lambda$. In other words, when $\sigma\left(b_{k}, a\right)$ becomes lower. According to such arguments, when using the ascending rule: 


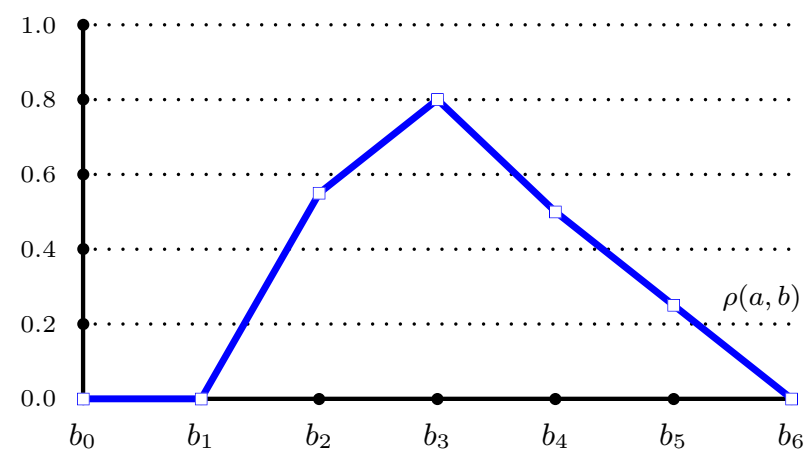

Figure 1: The min selecting function (consider action $a_{4}$ from Example 1)

- the selection of $C_{k+1}$ is justified if and only if $\sigma\left(a, b_{k+1}\right)>\sigma\left(b_{k}, a\right)$;

- on the contrary, if $\sigma\left(b_{k}, a\right) \geq \sigma\left(a, b_{k+1}\right)$, then the selection of $C_{k}$ is justified.

The min selecting function (3.2) leads precisely to the same selection as analyzed above for the ascending rule for the following reasons:

- according to $b$ ) of Corollary 3 (see Appendix A.1), $C_{k+1}$ is selected if and only if $\sigma\left(a, b_{k+1}\right)>\sigma\left(b_{k}, a\right)$ (the inequality $\sigma\left(b_{k}, a\right)<\sigma\left(b_{k+1}, a\right)$ being always verified, see the inequalities (3.4) above).

- according to $a$ ) of Lemma 1 (see Appendix A.1), $C_{k}$ is selected if and only if $\sigma\left(b_{k}, a\right) \geq \sigma\left(a, b_{k+1}\right)$ (the equality $\sigma\left(b_{k}, a\right)=\sigma\left(b_{k+1}, a\right)$ being excluded here, see the inequalities (3.4) above).

Let us consider now Property 2. The min selecting function (3.2) fulfills such a Property 2 (see Proof in Appendix A.1). If this property could not be fulfilled, then a better category could be selected for an action $a^{\prime}$ than another action $a$ that strictly dominates $a^{\prime}$.

It would be interesting to study other specific selecting functions too, but for the sake of simplicity, the min selecting function (3.2) seemed to us as a good choice. Let us notice that this specific function is also, for instance, used in the CLOSORT method proposed by Fernández et al. (2008). Nevertheless, these two methods are different in several aspects, as follows:

- Electre Tri-C is applied to the decision aiding sorting context where the categories are completely ordered, while CLOSORT was mainly designed to be applied where the categories are non-ordered.

- The min selecting function plays a slightly different role in both methods. In ELECTRE TRI-C such a function is used in each one of the joint rules to select one category between two consecutive categories, while in CLOSoRT the function is used to select one or several categories such that $\min \left\{\sigma\left(a, b_{h}\right), \sigma\left(b_{h}, a\right)\right\} \geq 0.50$, where $b_{h}$ in $B$ is the preferentially closest reference action to $a$.

- When using CLOSORT, the assignment of an action is only possible if the value of the overall min selecting function is at least equal to 0.50, while ELECTRE TRI-C provides always a range of at least one category.

- EleCtre Tri-C was designed according to some requirements such that indifference and incomparability situations are taken into account by proposing a range of categories likely to receive an action, while the final assignment results of CLOSORT are mainly provided by only one category per action (several breaking rules are used in case of ties).

\section{Properties of the ElECTRE TRI-C method}

The aim of this section is to analyze the properties of the ELECTRE TRI-C method based on the structural requirements defined in Section 3.1 and according to the conditions imposed to the set of characteristic actions, $B$.

According to the separability conditions introduced in Section 3.1, the minimum required level of credibility with respect to the characteristic actions, $\lambda^{b}$, is defined as follows:

$$
\lambda^{b}=\max _{h=1, \ldots,(q-1)}\left\{\sigma\left(b_{h}, b_{h+1}\right)\right\} .
$$


If the hyper-strict separability condition is fulfilled, then $\lambda^{b}=0$; if the strict separability condition is fulfilled, then $\lambda^{b} \in\left[0, \frac{1}{2}\left[\right.\right.$; and if the weak separability condition is fulfilled, then $\lambda^{b} \in[0,1[$.

\section{Theorem 1}

a) The conformity property holds if $\lambda>\lambda^{b}$.

b) The homogeneity, the monotonicity, and the stability properties hold.

Corollary 1 If the strict separability condition is fulfilled, then the conformity property holds.

Proofs of Theorem 1 and Corollary 1 are provided in Appendices A.2 and A.3, respectively. If the conformity property does not hold, then the decision aiding assignment model becomes inconsistent and, therefore, it is not able to support the assignment of the characteristic actions as well as the potential actions. According to such proofs, let us highlight the two following aspects:

i) after applying a merging operation, one necessarily has $\lambda>\lambda^{b^{\prime}}$, where $\lambda^{b^{\prime}}$ is the minimum required credibility level associated with $B^{\prime}$.

ii) after a splitting operation, the conformity property is verified only if $\lambda>\lambda^{b^{\prime}}$. If this last condition is not fulfilled, then the characteristic actions defined in the splitting operation should be redefined in order to verify the conformity property.

Let $\lambda$ be the chosen credibility level used to define the $\lambda$-binary relations (see Definition 1, in Section 2). Proposition 1 presents an important result in order to improve the interpretation of the results provided by Electre Tri-C.

Proposition 1 For any action a compared to the characteristic actions $b_{h}$ one and only one of the three following cases occurs:

a) Action $a$ is neither $\lambda$-indifferent nor $\lambda$-incomparable to $b_{h}$, for all $b_{h} \in B$.

b) Action a is $\lambda$-indifferent to at least one characteristic action $b_{h}$. Moreover, if $b_{h}$ is not unique, then the characteristic actions, which are $\lambda$-indifferent to action a, define a subset of consecutive categories.

c) Action a is $\lambda$-incomparable to at least one characteristic action $b_{h}$. Moreover, if $b_{h}$ is not unique, then the characteristic actions, which are $\lambda$-incomparable to action a, define a subset of consecutive categories.

Proof of Proposition 1 is trivial since when an action $a$ is compared to the boundary actions in ELECTRE TRI-B (see Section 6) it was proved that one and only one of three cases can occur (Roy and Bouyssou 1993, Rés. 6.3.1, p. 392). This result is still valid for ELECTRE TRI-C, since these two methods use the same outranking credibility indices and the chosen credibility level plays the same role (at least in a first step).

From each one of the two joint rules of the ElECTRE TRI-C method (Definitions 4 and 5), a single category is selected for a possible assignment of an action $a$. According to Theorem 2, the two selected categories can be distinct.

\section{Theorem 2}

a) The same category or two consecutive categories are selected by both joint rules, for a possible assignment of action a, when the case of Proposition 1.a) occurs.

b) The highest category, $C_{t}$, such that a is $\lambda$-indifferent to $b_{t}$, or the best adjacent category, $C_{t+1}$, is selected by the descending rule; and the lowest category, $C_{k}$, such that a is $\lambda$-indifferent to $b_{k}$, or to the worst adjacent category, $C_{k-1}$, is selected by the ascending rule, for a possible assignment of action a, when the case of Proposition 1.b) occurs.

c) The lowest category, $C_{t}$, such that a is $\lambda$-incomparable to $b_{t}$, or the worst adjacent category, $C_{t-1}$, is selected by the descending rule; and the highest category, $C_{k}$, such that a is $\lambda$-incomparable to $b_{k}$, or to the best adjacent category, $C_{k+1}$, is selected by the ascending rule, for a possible assignment of action a, when the case of Proposition 1.c) occurs. 
Proof of Theorem 2 is provided in Appendix A.4. From Theorem 2.b), a range of categories $\left[C_{h-1}, C_{h+1}\right]$ can be selected for a possible assignment of an action $a$, which is $\lambda$-indifferent to a unique $b_{h}$, according to a chosen credibility level, $\lambda$, and the chosen function $\rho(a, b)$. This means that the outranking credibility between $a$ and $b_{h}$, or $b_{h}$ and $a$ is weakly supported by the chosen credibility level, $\lambda$ (see also Definition 1).

Corollary 2 There is a credibility level, $\lambda^{a}$, such that the highest category, $C_{t}$, where a is $\lambda^{a}$-indifferent to $b_{t}$, is selected by the descending rule, and the lowest category, $C_{k}$, where a is $\lambda^{a}$-indifferent to $b_{k}$, is selected by the ascending rule, for a possible assignment of action a.

Proof of Corollary 2 is provided in Appendix A.5.

Let $\Gamma^{\lambda}(a)$ denote the subset of possible categories to assign an action $a$, which contains the lowest category and the highest category and all the intermediate categories (if they exist). The relationship between the chosen credibility level, $\lambda$, and the number of categories in $\Gamma^{\lambda}(a)$ is provided by Proposition 2 .

Proposition 2 If the chosen credibility level, $\lambda$, increases:

a) If one of the cases of Propositions 1.a) and 1.c) occurs, then the number of categories in $\Gamma^{\lambda}(a)$ will not decrease.

b) If the case of Proposition 1.b) occurs, then the number of categories in $\Gamma^{\lambda}(a)$ will not increase until at least one $\lambda$-indifference exists.

Proof of Proposition 2 is provided in Appendix A.6.

\section{$5 \quad$ Numerical examples}

This section presents two numerical examples in order to illustrate the theoretical results provided by the ELECTRE TRI-C method.

\subsection{Example 1}

Consider fifteen potential actions, denoted $a_{1}, \ldots, a_{15}$ evaluated on a coherent family of seven criteria, denoted $F=\left\{g_{1}, \ldots, g_{7}\right\}$ (all the criteria are in increasing preference direction), when taking into account the preferences of the decision maker (see Table 1). The power of each criterion is defined by the weights and the veto thresholds. This power is also presented in this table, but without considering veto thresholds.

Table 1: Criteria and parameters

\begin{tabular}{cccccccc}
\hline & \multicolumn{2}{c}{ Possible performances } & & \multicolumn{4}{c}{ Parameters } \\
\cline { 2 - 3 } \cline { 5 - 7 } Criteria & Worst & Best & & $q_{j}$ & $p_{j}$ & $v_{j}$ & $w_{j}$ \\
\hline$g_{1}$ & 0 & 50 & & 4 & 8 &. & 0.20 \\
$g_{2}$ & 0 & 100 & & 10 & 15 &. & 0.15 \\
$g_{3}$ & 0 & 100 & & 10 & 15 &. & 0.10 \\
$g_{4}$ & 0 & 20 & & 2 & 4 &. & 0.10 \\
$g_{5}$ & 0 & 20 & & 2 & 4 &. & 0.10 \\
$g_{6}$ & 0 & 6 & & 0 & 1 &. & 0.15 \\
$g_{7}$ & 0 & 6 & & 0 & 1 &. & 0.20 \\
\hline
\end{tabular}

The objective is to assign the potential actions (see Table 2) to a set of five categories, denoted $\left\{C_{1}, \ldots, C_{5}\right\}$, defined by a set of characteristic actions, denoted $B=\left\{b_{0}, b_{1}, \ldots, b_{5}, b_{6}\right\}$, according to a particular decision aiding sorting context (see Table 3). This set of characteristic actions fulfills the strict separability condition since $\lambda^{b}=0.30$. Therefore, the chosen credibility level can be any value within the range $[0.50,1]$.

A graphical representation of the characteristic actions and four potential actions (see Figure 2) led us to present the following remarks (whatever the chosen credibility level, $\lambda$, and the chosen vector of weights, $w_{j}, j=$ $1, \ldots, 7)$ : action $a_{2}$ can only be assigned to $C_{5}$; action $a_{15}$ can be assigned to $C_{3}$ or $C_{4}$; action $a_{9}$ can be assigned to $C_{1}$ or $C_{2}$; and action $a_{4}$ can be assigned to $C_{2}, C_{3}$, or $C_{4}$. 
Table 2: Potential actions

\begin{tabular}{crrrrrrr}
\hline Actions & $g_{1}$ & $g_{2}$ & $g_{3}$ & $g_{4}$ & $g_{5}$ & $g_{6}$ & $g_{7}$ \\
\hline$a_{1}$ & 16 & 15 & 40 & 12 & 15 & 5 & 3 \\
$a_{2}$ & 45 & 92 & 85 & 16 & 16 & 5 & 5 \\
$a_{3}$ & 21 & 62 & 24 & 16 & 12 & 5 & 3 \\
$a_{4}$ & 21 & 25 & 50 & 10 & 12 & 3 & 5 \\
$a_{5}$ & 4 & 12 & 15 & 4 & 2 & 2 & 1 \\
$a_{6}$ & 5 & 30 & 60 & 10 & 15 & 1 & 2 \\
$a_{7}$ & 6 & 25 & 25 & 4 & 16 & 4 & 5 \\
$a_{8}$ & 40 & 80 & 60 & 16 & 12 & 4 & 5 \\
$a_{9}$ & 10 & 20 & 30 & 8 & 8 & 2 & 1 \\
$a_{10}$ & 21 & 19 & 80 & 18 & 16 & 4 & 2 \\
$a_{11}$ & 10 & 4 & 47 & 11 & 15 & 4 & 2 \\
$a_{12}$ & 45 & 85 & 85 & 15 & 15 & 5 & 5 \\
$a_{13}$ & 15 & 16 & 72 & 15 & 18 & 4 & 2 \\
$a_{14}$ & 18 & 20 & 47 & 12 & 14 & 4 & 4 \\
$a_{15}$ & 35 & 70 & 60 & 10 & 10 & 3 & 3 \\
\hline Best $g_{j}(a)$ & 45 & 92 & 85 & 16 & 18 & 5 & 5 \\
Worst $g_{j}(a)$ & 4 & 4 & 15 & 2 & 2 & 1 & 1 \\
\hline
\end{tabular}

Table 3: Characteristic actions

\begin{tabular}{rrrrrrrrr}
\hline & & \multicolumn{7}{c}{ Criteria } \\
\cline { 3 - 9 }$C_{h}$ & $b_{h}$ & $g_{1}$ & $g_{2}$ & $g_{3}$ & $g_{4}$ & $g_{5}$ & $g_{6}$ & $g_{7}$ \\
\hline & $b_{0}$ & 0 & 0 & 0 & 0 & 0 & 0 & 0 \\
$C_{1}$ & $b_{1}$ & 5 & 10 & 20 & 5 & 5 & 1 & 1 \\
$C_{2}$ & $b_{2}$ & 15 & 30 & 40 & 10 & 10 & 2 & 2 \\
$C_{3}$ & $b_{3}$ & 25 & 50 & 60 & 10 & 10 & 3 & 3 \\
$C_{4}$ & $b_{4}$ & 35 & 70 & 60 & 10 & 10 & 4 & 4 \\
$C_{5}$ & $b_{5}$ & 45 & 90 & 80 & 15 & 15 & 5 & 5 \\
& $b_{6}$ & 50 & 100 & 100 & 20 & 20 & 6 & 6 \\
\hline
\end{tabular}

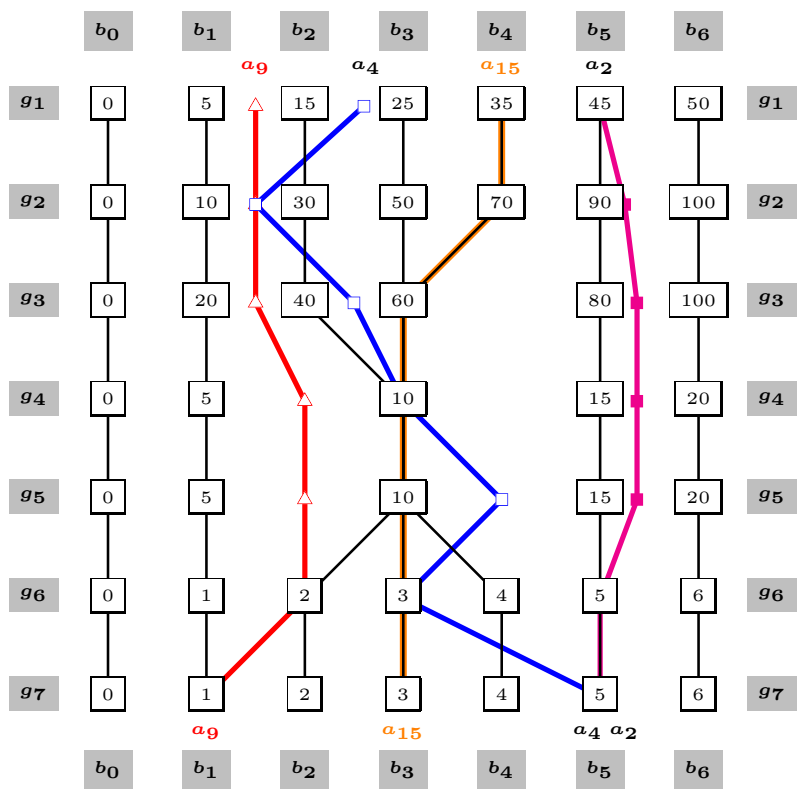

Figure 2: Representation of the characteristic actions

The credibility indices of the comprehensive outranking of the potential actions over the characteristic actions, and vice-versa, are presented in Table 4 . Let us choose two credibility levels: $\lambda_{1}=0.60$ and $\lambda_{2}=0.70$. Therefore, the results provided by Electre TrI-C (when using the min selecting function (3.2)) are presented in Table 5. 
Table 4: Outranking credibility (potential actions)

\begin{tabular}{|c|c|c|c|c|c|c|c|c|c|c|c|c|c|c|}
\hline \multirow[b]{2}{*}{ Actions } & \multicolumn{7}{|c|}{$\sigma\left(a, b_{h}\right)$} & \multicolumn{7}{|c|}{$\sigma\left(b_{h}, a\right)$} \\
\hline & $b_{0}$ & $b_{1}$ & $b_{2}$ & $b_{3}$ & $b_{4}$ & $b_{5}$ & $b_{6}$ & $b_{0}$ & $b_{1}$ & $b_{2}$ & $b_{3}$ & $b_{4}$ & $b_{5}$ & $b_{6}$ \\
\hline$a_{1}$ & 1.00 & 1.00 & 0.85 & 0.55 & 0.35 & 0.30 & 0.00 & 0.00 & 0.15 & 0.55 & 0.75 & 0.75 & 1.00 & 1.00 \\
\hline$a_{2}$ & 1.00 & 1.00 & 1.00 & 1.00 & 1.00 & 1.00 & 0.30 & 0.00 & 0.00 & 0.00 & 0.00 & 0.00 & 1.00 & 1.00 \\
\hline$a_{3}$ & 1.00 & 1.00 & 0.90 & 0.90 & 0.50 & 0.30 & 0.00 & 0.00 & 0.10 & 0.30 & 0.69 & 0.75 & 1.00 & 1.00 \\
\hline$a_{4}$ & 1.00 & 1.00 & 1.00 & 0.85 & 0.50 & 0.25 & 0.00 & 0.00 & 0.00 & 0.55 & 0.80 & 0.80 & 1.00 & 1.00 \\
\hline$a_{5}$ & 1.00 & 0.95 & 0.15 & 0.00 & 0.00 & 0.00 & 0.00 & 0.39 & 0.85 & 1.00 & 1.00 & 1.00 & 1.00 & 1.00 \\
\hline$a_{6}$ & 1.00 & 1.00 & 0.65 & 0.30 & 0.30 & 0.10 & 0.00 & 0.15 & 0.35 & 0.80 & 0.90 & 0.90 & 1.00 & 1.00 \\
\hline$a_{7}$ & 1.00 & 1.00 & 0.60 & 0.45 & 0.45 & 0.30 & 0.00 & 0.10 & 0.40 & 0.55 & 0.55 & 0.70 & 1.00 & 1.00 \\
\hline$a_{8}$ & 1.00 & 1.00 & 1.00 & 1.00 & 1.00 & 0.65 & 0.00 & 0.00 & 0.00 & 0.10 & 0.20 & 0.65 & 1.00 & 1.00 \\
\hline$a_{9}$ & 1.00 & 1.00 & 0.75 & 0.20 & 0.20 & 0.00 & 0.00 & 0.00 & 0.70 & 1.00 & 1.00 & 1.00 & 1.00 & 1.00 \\
\hline$a_{10}$ & 1.00 & 1.00 & 0.97 & 0.65 & 0.45 & 0.30 & 0.10 & 0.00 & 0.15 & 0.45 & 0.55 & 0.70 & 0.95 & 1.00 \\
\hline$a_{11}$ & 1.00 & 1.00 & 0.80 & 0.39 & 0.39 & 0.10 & 0.00 & 0.15 & 0.30 & 0.75 & 0.75 & 0.90 & 1.00 & 1.00 \\
\hline$a_{12}$ & 1.00 & 1.00 & 1.00 & 1.00 & 1.00 & 1.00 & 0.15 & 0.00 & 0.00 & 0.00 & 0.00 & 0.00 & 1.00 & 1.00 \\
\hline$a_{13}$ & 1.00 & 1.00 & 0.88 & 0.45 & 0.45 & 0.30 & 0.10 & 0.00 & 0.15 & 0.55 & 0.61 & 0.76 & 0.95 & 1.00 \\
\hline$a_{14}$ & 1.00 & 1.00 & 1.00 & 0.64 & 0.59 & 0.15 & 0.00 & 0.00 & 0.15 & 0.55 & 0.55 & 0.90 & 1.00 & 1.00 \\
\hline$a_{15}$ & 1.00 & 1.00 & 1.00 & 1.00 & 0.65 & 0.00 & 0.00 & 0.00 & 0.00 & 0.20 & 0.65 & 1.00 & 1.00 & 1.00 \\
\hline
\end{tabular}

Table 5: Electre TRI-C assignment results

\begin{tabular}{|c|c|c|c|c|c|c|c|c|}
\hline \multirow[b]{2}{*}{ Actions } & \multicolumn{4}{|c|}{$\lambda_{1}=0.60$} & \multicolumn{4}{|c|}{$\lambda_{2}=0.70$} \\
\hline & Nr. $I^{\lambda}$ & Nr. $R^{\lambda}$ & $\begin{array}{l}\text { Lowest } \\
\text { Category }\end{array}$ & $\begin{array}{l}\text { Highest } \\
\text { Category }\end{array}$ & Nr. $I^{\lambda}$ & Nr. $R^{\lambda}$ & $\begin{array}{c}\text { Lowest } \\
\text { Category }\end{array}$ & $\begin{array}{l}\text { Highest } \\
\text { Category }\end{array}$ \\
\hline$a_{1}$ & 0 & 0 & $C_{2}$ & $C_{3}$ & 0 & 0 & $C_{2}$ & $C_{3}$ \\
\hline$a_{2}$ & 1 & 0 & $C_{5}$ & $C_{5}$ & 1 & 0 & $C_{5}$ & $C_{5}$ \\
\hline$a_{3}$ & 1 & 0 & $C_{3}$ & $C_{3}$ & 0 & 0 & $C_{3}$ & $C_{3}$ \\
\hline$a_{4}$ & 1 & 0 & $C_{3}$ & $C_{3}$ & 1 & 0 & $C_{3}$ & $C_{3}$ \\
\hline$a_{5}$ & 1 & 0 & $C_{1}$ & $C_{1}$ & 1 & 0 & $C_{1}$ & $C_{1}$ \\
\hline$a_{6}$ & 1 & 0 & $C_{2}$ & $C_{2}$ & 0 & 0 & $C_{2}$ & $C_{2}$ \\
\hline$a_{7}$ & 0 & 1 & $C_{2}$ & $C_{3}$ & 0 & 2 & $\mathrm{C}_{2}$ & $C_{3}$ \\
\hline$a_{8}$ & 2 & 0 & $C_{4}$ & $C_{5}$ & 0 & 0 & $C_{4}$ & $C_{5}$ \\
\hline$a_{9}$ & 2 & 0 & $C_{1}$ & $C_{2}$ & 2 & 0 & $C_{1}$ & $C_{2}$ \\
\hline$a_{10}$ & 0 & 0 & $C_{3}$ & $C_{3}$ & 0 & 1 & $C_{3}$ & $C_{3}$ \\
\hline$a_{11}$ & 1 & 0 & $C_{2}$ & $C_{2}$ & 1 & 0 & $C_{2}$ & $C_{2}$ \\
\hline$a_{12}$ & 1 & 0 & $C_{5}$ & $C_{5}$ & 1 & 0 & $C_{5}$ & $C_{5}$ \\
\hline$a_{13}$ & 0 & 0 & $C_{2}$ & $C_{2}$ & 0 & 1 & $\mathrm{C}_{2}$ & $C_{3}$ \\
\hline$a_{14}$ & 0 & 0 & $C_{4}$ & $C_{4}$ & 0 & 1 & $C_{3}$ & $C_{4}$ \\
\hline$a_{15}$ & 2 & 0 & $C_{3}$ & $C_{4}$ & 0 & 0 & $C_{3}$ & $C_{4}$ \\
\hline
\end{tabular}

According to the assignment results of ELECTRE TRI-C presented in Table 5, let us observe the behavior of three $\lambda$-binary relations:

1) There is only $\lambda$-preference:

- the category $C_{h}$ is conjointly selected to assign action $a$, where $a P^{\lambda} b_{h}$ and $b_{h+1} P^{\lambda} a$. When $\lambda=0.60$, see the assignment of $a_{10}$ and $a_{13}$. When $\lambda=0.70$, see the assignment of $a_{3}$;

- the category $C_{h}$ is conjointly selected to assign action $a$, where $a P^{\lambda} b_{h-1}$ and $b_{h} P^{\lambda} a$. When $\lambda=0.60$, see the assignment of $a_{14}$. When $\lambda=0.70$, see the assignment of $a_{6}$;

- the range of categories $\left[C_{h}, C_{h+1}\right]$ is given for a possible assignment of action $a$, where $a P^{\lambda} b_{h}$ and $b_{h+1} P^{\lambda} a$. When $\lambda=0.60$, see the assignment of $a_{1}$. When $\lambda=0.70$, see the assignment of $a_{1}, a_{8}$, and $a_{15}$.

2) There is at least one $\lambda$-indifference:

- the category $C_{h}$ is conjointly selected to assign action $a$, where $a I^{\lambda} b_{h}$. When $\lambda=0.60$, see the assignment of $a_{2}, a_{3}, a_{4}, a_{5}, a_{6}, a_{11}$, and $a_{12}$. When $\lambda=0.70$, see the assignment of $a_{2}, a_{4}, a_{5}, a_{11}$, and $a_{12}$;

- the range of categories $\left[C_{h}, C_{h+1}\right]$ is given for a possible assignment of action $a$, where $a I^{\lambda} b_{h}$ and $a I^{\lambda} b_{h+1}$. When $\lambda=0.60$, see the assignment of $a_{8}, a_{9}$, and $a_{15}$. When $\lambda=0.70$, see the assignment of $a_{9}$. Noticed that since $a_{9}$ strictly dominates $b_{1}, b_{2}$ strictly dominates $a_{9}$, and $b_{2}$ is strictly separable from $b_{1}$, one obtains $a_{9} I^{\lambda} b_{1}$ and $a_{9} I^{\lambda} b_{2}$. This is a very particular case to achieve two $\lambda$-indifference relations. 
3) There is at least one $\lambda$-incomparability:

- the category $C_{h}$ is conjointly selected to assign action $a$, where $a R^{\lambda} b_{h}$. When $\lambda=0.70$, see the assignment of $a_{10}$;

- the range of categories $\left[C_{h-1}, C_{h}\right]$ is given for a possible assignment of action $a$, where $a R^{\lambda} b_{h}$. When $\lambda=0.60$, see the assignment of $a_{7}$. When $\lambda=0.70$, see the assignment of $a_{13}$;

- the range of categories $\left[C_{h}, C_{h+1}\right]$ is given for a possible assignment of action $a$, where $a R^{\lambda} b_{h}$. When $\lambda=0.70$, see the assignment of $a_{14}$;

- the range of categories $\left[C_{h}, C_{h+1}\right]$ is given for a possible assignment of action $a$, where $a R^{\lambda} b_{h}$ and $a R^{\lambda} b_{h+1}$. When $\lambda=0.70$, see the assignment of $a_{7}$.

These numerical results of ELECTRE TRI-C illustrate the theoretical results provided by Theorems 1 and 2 , Corollaries 1 and 2, as well as Propositions 1 and 2.

\section{$5.2 \quad$ Example 2}

This numerical example is based on a real-world decision aiding problem analyzed for a private infertility centre in Lisbon (Portugal), which the aim is to help infertile couples (women/men) to become parents while avoiding multiple pregnancies when using Assisted Reproductive Technology (Matias 2008). In such an application, the couples were evaluated on a set of seven criteria, co-constructed through an interaction process between the analyst and the medical experts. For this numerical example, only the preferences of the embryologist is considered, but in the original application the medical experts were also formed by gynaecologists/obstetricians.

The objective of the decision aiding sorting model is to give a "recommendation" to the embryologist about the number of embryos to transfer to the uterus of the women in order to obtain a pregnancy and, at the same time, to reduce the risk of multiple pregnancies. Of course the final decision should be made by the couples themselves, but the medical experts "recommendation" is very important. The nature of the set of categories is related to the transfer of embryos: four embryos $\left(C_{1}\right)$, three embryos $\left(C_{2}\right)$, two embryos $\left(C_{3}\right)$, and one embryo $\left(C_{4}\right)$. These categories are clearly ordered from the least risky category, $C_{4}$, to the most risky category, $C_{1}$. The set of categories was defined by characteristic actions.

For this numerical example, a subset of twenty five couples, denoted $a_{1}, \ldots, a_{25}$, was randomly selected from a total of fifty three to be assigned to the set of four embryo-transfert categories. The chosen credibility level accepted by the medical experts was $\lambda=0.60$. Therefore, the ElECTRE TRI-C assignment results (when using the min selecting function (3.2)) and the real medical treatments are presented in Table 6.

Table 6: EleCtre TrI-C versus medical expert's results

\begin{tabular}{cccccc}
\hline & \multicolumn{5}{c}{ ELECTRE TRI-C assignments } \\
\cline { 2 - 5 } Couples & Nr. $I^{\lambda}$ & Nr. $R^{\lambda}$ & Lowest category & Highest category & Medical expert \\
\hline$a_{1}$ & 0 & 1 & $C_{1}$ & $C_{2}$ & $C_{2}$ \\
$a_{2}$ & 0 & 0 & $C_{2}$ & $C_{2}$ & $C_{2}$ \\
$a_{3}$ & 0 & 0 & $C_{3}$ & $C_{3}$ & $C_{3}$ \\
$a_{4}$ & 0 & 0 & $C_{3}$ & $C_{3}$ & $C_{3}$ \\
$a_{5}$ & 0 & 0 & $C_{2}$ & $C_{2}$ & $C_{2}$ \\
$a_{6}$ & 0 & 0 & $C_{3}$ & $C_{3}$ & $C_{3}$ \\
$a_{7}$ & 0 & 0 & $C_{2}$ & $C_{2}$ & $C_{2}$ \\
$a_{8}$ & 1 & 0 & $C_{3}$ & $C_{3}$ & $C_{3}$ \\
$a_{9}$ & 0 & 0 & $C_{3}$ & $C_{3}$ & $C_{3}$ \\
$a_{10}$ & 0 & 0 & $C_{3}$ & $C_{3}$ & $C_{3}$ \\
$a_{11}$ & 0 & 0 & $C_{3}$ & $C_{3}$ & $C_{3}$ \\
$a_{12}$ & 0 & 1 & $C_{3}$ & $C_{3}$ & $C_{3}$ \\
$a_{13}$ & 0 & 0 & $C_{3}$ & $C_{3}$ & $C_{3}$ \\
$a_{14}$ & 1 & 0 & $C_{3}$ & $C_{3}$ & $C_{2}$ \\
$a_{15}$ & 0 & 0 & $C_{2}$ & $C_{2}$ & $C_{3}$ \\
$a_{16}$ & 1 & 0 & $C_{3}$ & $C_{3}$ & $C_{3}$ \\
$a_{17}$ & 1 & 0 & $C_{3}$ & $C_{3}$ & $C_{3}$ \\
$a_{18}$ & 0 & 0 & $C_{3}$ & $C_{3}$ & $C_{3}$ \\
$a_{19}$ & 0 & 1 & $C_{3}$ & $C_{3}$ & $C_{3}$ \\
$a_{20}$ & 0 & 0 & $C_{3}$ & $C_{3}$ & $C_{3}$ \\
$a_{21}$ & 1 & 0 & $C_{3}$ & $C_{3}$ & $C_{3}$ \\
$a_{22}$ & 0 & 0 & $C_{3}$ & $C_{3}$ & $C_{3}$ \\
$a_{23}$ & 1 & 0 & $C_{3}$ & $C_{3}$ & $C_{3}$ \\
$a_{24}$ & 1 & 0 & $C_{3}$ & $C_{3}$ & $C_{2}$ \\
$a_{25}$ & 0 & 0 & $C_{2}$ & $C_{2}$ & \\
\hline
\end{tabular}

Source: Data and more details in Matias (2008). 
According to the assignment results presented in Table 6, the sorting accuracy (when ELECTRE TRI-C provides a single category, which is the same as provided by the medical experts) is $96 \%$. In this numerical example, only the the medical treatment of the couple $a_{1}$ was not exactly the same as the "recommendation" provided by Electre Tri-C. This particular issue allows to extend the usefulness of such a new sorting method.

\section{Electre Tri-B versus Electre Tri-C}

The aim of this section is to present the Electre TrI-B method and a comparison with Electre TrI-C regarding their assumptions, requirements, strengths, assignment procedures, and so on, in order to help potential users to make an informed choice between these two methods.

Electre Tri-C was conceived to be used in decision aiding sorting contexts where EleCtre Tri-B does not fit well the problem statement or if it seems difficult to define boundary actions according to the Assumption 4 (see Section 1). Defining boundary actions is often a very hard task. This is particularly the case when the decision maker has a fuzzy idea of the boundary between two consecutive categories. In many real-world decision aiding situations (see the examples mentioned in the Introduction section) these frontiers have no objective existence, since the separation between two consecutive categories can be conceived in several different ways.

According to $\mathrm{Yu}$ (1992) and Roy and Bouyssou (1993, p. 389-401), when using ElECTRE TrI-B, an action $a$ is compared to the boundary actions, which define the set of categories, denoted here $\left\{\widehat{C}_{1}, \ldots, \widehat{C}_{h}, \ldots, \widehat{C}_{q}\right\}$ where $\widehat{C}_{1}$ is the worst category and $\widehat{C}_{q}$ is the best one, with $q \geq 2$. The categories are defined by boundary actions, denoted here $\hat{b}_{1}, \ldots, \hat{b}_{q}, \hat{b}_{q+1}$. These actions define the frontiers of each one of the categories. By convention, for $h=1, \ldots, q$, the lower boundary action, $\hat{b}_{h}$, belongs to $\widehat{C}_{h}$. Consequently, its upper boundary action, $\hat{b}_{h+1}$, belongs to $\widehat{C}_{h+1}$ (categories are closed from below) (see Figure 3 ).

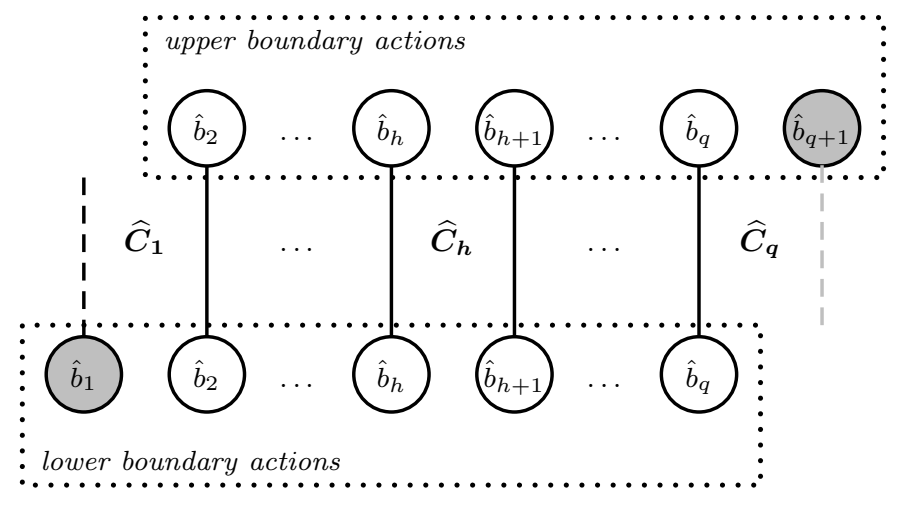

Figure 3: Definition of boundary actions

Let $\widehat{B}=\left\{\hat{b}_{1}, \hat{b}_{2}, \ldots, \hat{b}_{h}, \ldots, \hat{b}_{q}, \hat{b}_{q+1}\right\}$ denote the set of the $(q+1)$ boundary actions. This set of boundary actions must fulfill at least the weak separability (Condition 1). Furthermore, the role played by $\hat{b}_{1}$ and $\hat{b}_{q+1}$, when using boundary actions, is the same as $b_{0}$ and $b_{q+1}$ with the presence of characteristic actions.

Two assignment procedures of EleCtre TRI-B were originally proposed. In order to distinguish them, the authors were qualified one of them by pessimistic and the other one by optimistic. Roy (2002) showed that such two concepts could lead to misunderstanding and taking into account the nature of these procedures, it would be suitable to replace pessimistic by pseudo-conjunctive and optimistic by pseudo-disjunctive. Such two assignment procedures have been mainly used separately and they can be rewritten as follows:

Definition 7 (Pseudo-conjunctive assignment procedure) Choose a credibility level, $\lambda\left(\frac{1}{2} \leq \lambda \leq 1\right)$. Decrease $h$ from $(q+1)$ until the first value, $t$, such that $\sigma\left(a, \hat{b}_{t}\right) \geq \lambda$. Assign action a to category $\widehat{C}_{t}$.

Definition 8 (Pseudo-disjunctive assignment procedure) Choose a credibility level, $\lambda\left(\frac{1}{2} \leq \lambda \leq 1\right)$. Increase $h$ from 1 until the first value, $k$, such that $\sigma\left(\hat{b}_{k}, a\right) \geq \lambda$ and $\sigma\left(a, \hat{b}_{k}\right)<\lambda$. Assign action a to category $\widehat{C}_{k-1}$.

Electre Tri-C fulfills similar properties as Electre Tri-B (see Roy and Bouyssou 1993, p. 394-6). When using boundary actions such properties must be formulated in an appropriate way in order to fit the problem 
statement (see Roy and Bouyssou 1993, p. 355, or Rogers et al. 2000, p. 78-9). For instance, the stability property may be formulated as follows: "when applying a merging or a splitting operation, the actions previously assigned to the non-modified categories will be assigned to the same categories, after modification." In such a case, the merging operation consists of removing an existing boundary action, and the splitting operation consists of adding a new boundary action.

Based on the ELECTRE TRI-B assignment procedures, if an action $a$ is assigned to category $\widehat{C}_{t}$ by the pseudoconjunctive rule and to $\widehat{C}_{k}$ by the pseudo-disjunctive rule, then it was proved that $t \leq k$ (see Roy and Bouyssou 1993, p. 394-6). Moreover, such two assignment procedures provide different results if and only if there is at least one $h$ such that $a R^{\lambda} \hat{b}_{h}$ (see Definition 1.d), in Section 2).

When using Electre TrI-B, the chosen credibility level is necessary and sufficient to validate the assignment of an action to a category based on the outranking credibility indices. Similarly, when using ElECTRE TrI-C the chosen credibility level is still necessary, but it is not sufficient because a function $\rho(a, b)$ is needed too.

Let us illustrate this idea by taking into account the role played by the reference actions in each one of these two methods on the descending-type rules. Consider an action $a$ and two reference actions, $b_{h}$ and $b_{h+1}$, which fulfill the strict separability (Condition 2), such that $g_{j}\left(b_{h}\right) \leq g_{j}(a)<g_{j}\left(b_{h+1}\right)$, for all $g_{j} \in F$. In such a case, $\sigma\left(a, b_{h}\right)=1$ and $\sigma\left(b_{h+1}, a\right)=1$. If $b_{h}$ and $b_{h+1}$ play the role of the lower and the upper bounds of category $\widehat{C}_{h}$, respectively, then action $a$ is necessarily assigned to $\widehat{C}_{h}$ by the pseudo-conjunctive rule of ELECTRE TRI-B. If $b_{h}$ and $b_{h+1}$ play the role of the characteristic actions of categories $C_{h}$ and $C_{h+1}$, respectively, then action $a$ can be assigned to $C_{h}$ or $C_{h+1}$ by the descending rule of ELECTRE TRI-C. In a general case, according to the definition of the reference action, $b_{h}$, a category $C_{h}$ in ELECTRE TRI-C can receive actions $a$ such that $a \lambda$-outranks $b_{h}$ or $a$ is $\lambda$-outranked by $b_{h}$, according to the descending rule. This is not possible when using ELECTRE TRI-B. Similarly analysis can be done for the ascending-type rules.

ELECTRE TRI-B is currently one of the most used sorting methods for dealing with sorting problems. Indeed, over the last few years, several applications have been modeled with ELECTRE TRI-B: accreditation system (Siskos et al. 2007), non-financial performance (André and Roy 2007), tourism industry (Roget and González 2005), zoning risk analysis (Merad et al. 2004), dangerous material transport (Costa et al. 2004), electricity market (Mavrotas et al. 2003), national priorities (Georgopoulou et al. 2003), climate change (Diakoulaki and Hontou 2003), public transport ticket system (Mousseau et al. 2001), land-use suitability assessment (Joerin et al. 2001), sustainable water resources (Raju et al. 2000), cropping systems (Arondel and Girardin 2000), and many others (it should be noticed that as for our best knowledge, there are several applications without publications).

\section{Conclusions}

This paper dealt with a new sorting method, called ElECTRE TRI-C, according to a decision aiding constructive approach. The pre-defined and ordered categories are defined through characteristic reference actions. The set of all characteristic actions should be co-constructed through an interactive process between the analyst and the decision maker taking into account the further similar analysis that the actions, which will be assigned to a category, should be subjected to. In our opinion, defining categories by using characteristic actions is of the utmost importance for modeling a wide variety of practical decision aiding situations dealing with sorting problems.

ElECTRE TRI-C is composed of two joint rules, called descending rule and ascending rule. Each one of these rules selects only one category for a possible assignment of an action. They are used conjointly in order to highlight the highest category and the lowest category, which can appear potentially appropriate to receive an action. These two extreme categories can be the same. When they differ, this means that the assignment of such an action remains ill-determined taking into account the way that the set of characteristic actions defines the categories.

It was proved in this paper that the ELECTRE TRI-C method fulfills the fundamental properties of conformity, homogeneity, monotonicity, and stability. There is a minimum required credibility level, which is associated with the definition of the characteristic actions in order to obtain a consistent decision aiding assignment model.

Since the veto assumptions are not required in all of the practical applications, the numerical example 1 (Section 5.1) does not make use of these assumptions. But, the veto effects were analyzed in the numerical example 2 (Section 5.2), where only the final assignment results were presented in order to illustrate a high sorting accuracy of the ELECTRE TRI-C method. Let us notice that a veto effect can reduce the degree of credibility of at least one of the four statements of the selecting function regarding the two consecutive categories. In such a case, the assignment of an action can be modified by this veto effect. 
Different variants, or extensions, of the outranking credibility indices have been proposed without changing their fundamental properties (see, for example, Figueira et al. 2009; Roy and Słowiński 2008). Such extensions take into account the effects of reinforced preference and counter-veto, and the interaction between criteria, respectively. The definitions and results presented in this paper remain valid when using these variants, or extensions.

Alternative selecting functions can also be suggested to replace the one proposed in this paper. Such alternative selecting functions must fulfill the two desired properties in order to preserve the role of the characteristic actions and the fundamental properties of the EleCTRE TRI-C method, in particular the monotonicity property. Therefore, the alternative selecting functions can increase, or decrease, the range of categories in the final assignment results, mainly due to the incomparability situations.

As for future research avenues, a decision support system incorporating the concept of characteristic actions is to be implemented. We also intend to analyze a possible extension to multiple characteristic actions. Such an extension will allow to model a larger number of decision aiding situations in the field of sorting problems. Currently there is no method incorporating the notion of category size in the sorting methods to limit the number of actions that can be assigned to each category. Indeed, we also intend to study this particular issue by introducing a notion of relative comprehensive comparison of the actions to define a new sorting problematic. At the same time, we should focus our attention on the inference of some parameters through an disaggregation-aggregation elicitation techniques using characteristic actions.

\section{Acknowledgements}

The authors would like to acknowledge the two anonymous referees for their helpful comments and suggestions. Juscelino Almeida DiAs acknowledges the financial support from the grant SFRH/ BD/ 22985/ 2005 (Fundação para a Ciência e a Tecnologia, Portugal). The authors also acknowledge the financial support from the Luso-French bilateral cooperation agreement between LAMSADE and CEG-IST (FCT/CNRS 2009). This research also partially benefited from the COST Action grant IC0602 on "Algorithmic Decision Theory".

\section{A Appendix: Proofs}

\section{A.1 Proof of Property 2 (in Section 3.2)}

Let $\rho(a, b)$ be a selecting function such that $\rho(a, b)=\min \{\sigma(a, b), \sigma(b, a)\}$. This proof is based on the following Lemma and Corollary.

\section{Lemma 1}

a) $\min \left\{\sigma\left(a, b_{h}\right), \sigma\left(b_{h}, a\right)\right\} \geq \min \left\{\sigma\left(a, b_{h+1}\right), \sigma\left(b_{h+1}, a\right)\right\}$ if and only if $\sigma\left(b_{h}, a\right) \geq \sigma\left(a, b_{h+1}\right)$ or $\sigma\left(b_{h}, a\right)=\sigma\left(b_{h+1}, a\right)$.

b) $\min \left\{\sigma\left(a, b_{h+1}\right), \sigma\left(b_{h+1}, a\right)\right\} \geq \min \left\{\sigma\left(a, b_{h}\right), \sigma\left(b_{h}, a\right)\right\}$ if and only if $\sigma\left(a, b_{h+1}\right) \geq \sigma\left(b_{h}, a\right)$ or $\sigma\left(a, b_{h+1}\right)=$ $\sigma\left(a, b_{h}\right)$.

\section{Proof of this Lemma:}

First, two mutually exclusive cases must successively be analyzed: $\rho\left(a, b_{h}\right)=\sigma\left(a, b_{h}\right)<\sigma\left(b_{h}, a\right)$ (case 1$)$ and $\rho\left(a, b_{h}\right)=\sigma\left(b_{h}, a\right) \leq \sigma\left(a, b_{h}\right)$ (case 2$)$. In each one of these two cases, let us examine in what conditions one has: $\rho\left(a, b_{h}\right) \geq \rho\left(a, b_{h+1}\right)$.

Case 1: $\rho\left(a, b_{h}\right)=\sigma\left(a, b_{h}\right)<\sigma\left(b_{h}, a\right)$.

According to the monotonicity of $\sigma(a, b)$ and $\sigma(b, a)$, one has $\sigma\left(a, b_{h+1}\right) \leq \sigma\left(a, b_{h}\right)<\sigma\left(b_{h}, a\right) \leq \sigma\left(b_{h+1}, a\right)$. Then, $\rho\left(a, b_{h+1}\right)=\sigma\left(a, b_{h+1}\right) \leq \sigma\left(a, b_{h}\right)$. In such a case, the inequality of $\left.a\right)$ of this Lemma is always verified.

Case 2: $\rho\left(a, b_{h}\right)=\sigma\left(b_{h}, a\right) \leq \sigma\left(a, b_{h}\right)$.

In such conditions, one has $\rho\left(a, b_{h}\right) \geq \rho\left(a, b_{h+1}\right)$ if and only if $\sigma\left(b_{h}, a\right) \geq \min \left\{\sigma\left(a, b_{h+1}, \sigma\left(b_{h+1}, a\right)\right)\right\}$. This is either equivalent to $\sigma\left(b_{h}, a\right) \geq \sigma\left(a, b_{h+1}\right)$ or to $\sigma\left(b_{h}, a\right) \geq \sigma\left(b_{h+1}, a\right)$. This last condition is equivalent 
to $\sigma\left(b_{h}, a\right)=\sigma\left(b_{h+1}, a\right)$ because $\sigma\left(b_{h+1}, a\right) \geq \sigma\left(b_{h}, a\right)$. Then, the inequality or the equality of $\left.a\right)$ of this Lemma is always verified.

Second, two another mutually exclusive cases must successively be analyzed: $\rho\left(a, b_{h+1}\right)=\sigma\left(a, b_{h+1}\right)<\sigma\left(b_{h+1}, a\right)$ (case 3) and $\rho\left(a, b_{h+1}\right)=\sigma\left(b_{h+1}, a\right) \leq \sigma\left(a, b_{h+1}\right)$ (case 4). In each one of these two cases, let us examine in what conditions one has: $\rho\left(a, b_{h+1}\right) \geq \rho\left(a, b_{h}\right)$.

Case 3: $\rho\left(a, b_{h+1}\right)=\sigma\left(a, b_{h+1}\right)<\sigma\left(b_{h+1}, a\right)$.

In such conditions, one has $\rho\left(a, b_{h+1}\right) \geq \rho\left(a, b_{h}\right)$ if and only if $\sigma\left(a, b_{h+1}\right) \geq \min \left\{\sigma\left(a, b_{h}, \sigma\left(b_{h}, a\right)\right)\right\}$. This is either equivalent to $\sigma\left(a, b_{h+1}\right) \geq \sigma\left(b_{h}, a\right)$ or to $\sigma\left(a, b_{h+1}\right) \geq \sigma\left(a, b_{h}\right)$. This last condition is equivalent to $\sigma\left(a, b_{h+1}\right)=\sigma\left(a, b_{h}\right)$ because $\sigma\left(a, b_{h}\right) \geq \sigma\left(a, b_{h+1}\right)$. Then, the inequality or the equality of $\left.b\right)$ of this Lemma is always verified.

Case 4: $\rho\left(a, b_{h+1}\right)=\sigma\left(b_{h+1}, a\right) \leq \sigma\left(a, b_{h+1}\right)$.

According to the monotonicity of $\sigma(a, b)$ and $\sigma(b, a)$, one has $\sigma\left(b_{h}, a\right) \leq \sigma\left(b_{h+1}, a\right) \leq \sigma\left(a, b_{h+1}\right) \leq \sigma\left(a, b_{h}\right)$. Then, $\rho\left(a, b_{h}\right)=\sigma\left(b_{h}, a\right) \leq \sigma\left(b_{h+1}, a\right)$. In such a case, the inequality of $\left.b\right)$ of this Lemma is always verified.

\section{Corollary 3}

a) $\min \left\{\sigma\left(a, b_{h}\right), \sigma\left(b_{h}, a\right)\right\}>\min \left\{\sigma\left(a, b_{h+1}\right), \sigma\left(b_{h+1}, a\right)\right\}$ if and only if $\sigma\left(b_{h}, a\right)>\sigma\left(a, b_{h+1}\right)$ and $\sigma\left(a, b_{h+1}\right)<$ $\sigma\left(a, b_{h}\right)$.

b) $\min \left\{\sigma\left(a, b_{h+1}\right), \sigma\left(b_{h+1}, a\right)\right\}>\min \left\{\sigma\left(a, b_{h}\right), \sigma\left(b_{h}, a\right)\right\}$ if and only if $\sigma\left(a, b_{h+1}\right)>\sigma\left(b_{h}, a\right)$ and $\sigma\left(b_{h}, a\right)<$ $\sigma\left(b_{h+1}, a\right)$.

\section{Proof of this Corollary:}

The proof of this Corollary is directly obtained from the proof of the above Lemma as follows:

i) The logic negation of $b$ ) of Lemma 1 is exactly the $a$ ) of this Corollary.

ii) The logic negation of $a$ ) of Lemma 1 is exactly the $b$ ) of this Corollary.

Consequently, the proof of Property 2 is stated as follows:

1) Case of the descending rule (Definition 4).

Let $C_{t}$ be the pre-selected category for action $a$ as well as for action $a^{\prime}$. This implies that $\sigma\left(a, b_{t}\right) \geq \lambda$ and $\sigma\left(a, b_{t+1}\right)<\lambda$ as well as $\sigma\left(a^{\prime}, b_{t}\right) \geq \lambda$ and $\sigma\left(a^{\prime}, b_{t+1}\right)<\lambda$. If for action $a$ one has $\rho\left(a, b_{t}\right)>\rho\left(a, b_{t+1}\right)$, then, according to $a$ ) of Corollary 3 , one has $\sigma\left(b_{t}, a\right)>\sigma\left(a, b_{t+1}\right)$. Taking into account that $a$ strictly dominates $a^{\prime}$, then according to the monotonicity of $\sigma(a, b)$ and $\sigma(b, a)$, one necessarily has $\sigma\left(b_{t}, a^{\prime}\right) \geq \sigma\left(b_{t}, a\right)$ and $\sigma\left(a, b_{t+1}\right) \geq \sigma\left(a^{\prime}, b_{t+1}\right)$. In such conditions, $\sigma\left(b_{t}, a^{\prime}\right)>\sigma\left(a^{\prime}, b_{t+1}\right)$. This implies that, according to $\left.a\right)$ of Corollary 3 , one obtains $\rho\left(a^{\prime}, b_{t}\right)>\rho\left(a^{\prime}, b_{t+1}\right)$.

2) Case of the ascending rule (Definition 5).

Let $C_{k+1}$ be the pre-selected category for action $a^{\prime}$ as well as for action $a$. This implies that $\sigma\left(b_{k+1}, a^{\prime}\right) \geq \lambda$ and $\sigma\left(b_{k}, a^{\prime}\right)<\lambda$ as well as $\sigma\left(b_{k+1}, a\right) \geq \lambda$ and $\sigma\left(b_{k}, a\right)<\lambda$. If for action $a^{\prime}$ one has $\rho\left(a^{\prime}, b_{k+1}\right)>\rho\left(a^{\prime}, b_{k}\right)$, then, according to $b$ ) of Corollary 3 , one has $\sigma\left(a^{\prime}, b_{k+1}\right)>\sigma\left(b_{k}, a^{\prime}\right)$. Taking into account that $a$ strictly dominates $a^{\prime}$, then according to the monotonicity of $\sigma(a, b)$ and $\sigma(b, a)$, one necessarily has $\sigma\left(b_{k}, a^{\prime}\right) \geq \sigma\left(b_{k}, a\right)$ and $\sigma\left(a, b_{k+1}\right) \geq \sigma\left(a^{\prime}, b_{k+1}\right)$. In such conditions, $\sigma\left(a, b_{k+1}\right)>\sigma\left(b_{k}, a\right)$. This implies that, according to $\left.b\right)$ of Corollary 3 , one obtains $\rho\left(a, b_{k+1}\right)>\rho\left(a, b_{k}\right)$. 


\section{A.2 Proof of Theorem 1 (in Section 4)}

This proof of Theorem 1 is applied to the descending rule. It remains valid for the ascending rule according to the transposition operation (see Definition 6, in Section 3.3).

a) Conformity:

Assume that the strict separability condition holds. Such a condition means that $\sigma\left(b_{h}, b_{h+1}\right)<\frac{1}{2}, h=$ $1, \ldots,(q-1)$. By definition of the characteristic actions and by construction of the credibility indices, one has $\sigma\left(b_{h+1}, b_{h}\right)=1, h=1, \ldots,(q-1)$ and $\sigma\left(b_{h}, b_{h}\right)=1, h=1, \ldots, q$. When applying the descending rule, $C_{t}$ is selected for the assignment of each characteristic action $b_{t}$ if and only if $\rho\left(b_{t}, b_{t}\right)>\rho\left(b_{t}, b_{t+1}\right)$. This condition is verified, since $\rho(b, b)$ fulfills Property 1 . The proof is similar when $\lambda^{b}<\lambda \leq 1$.

\section{b.1) Homogeneity:}

By definition two different actions, $a$ and $a^{\prime}$, are compared themselves in an identical manner with respect to the characteristic actions if and only if the following conditions are verified: $\sigma\left(a, b_{h}\right)=\sigma\left(a^{\prime}, b_{h}\right)$ and $\sigma\left(b_{h}, a\right)=\sigma\left(b_{h}, a^{\prime}\right)$, for all $h=1, \ldots, q$. Therefore, for each chosen credibility level, $\lambda$, the homogeneity property is verified because the selection of category $C_{t}$ for a possible assignment of action $a$ by the descending rule only depends on $\rho\left(a, b_{t}\right)=f\left(\sigma\left(a, b_{t}\right), \sigma\left(b_{t}, a\right)\right)$ and $\rho\left(a, b_{t+1}\right)=f\left(\sigma\left(a, b_{t+1}\right), \sigma\left(b_{t+1}, a\right)\right)$ or on $\rho\left(a, b_{t-1}\right)=f\left(\sigma\left(a, b_{t-1}\right), \sigma\left(b_{t-1}, a\right)\right)$ and $\rho\left(a, b_{t}\right)=f\left(\sigma\left(a, b_{t}\right), \sigma\left(b_{t}, a\right)\right)$.

b.2) Monotonicity:

From the monotonicity properties of $\sigma(a, b)$ and $\sigma(b, a)$, if an action $a$ strictly dominates action $a^{\prime}$, then $\sigma\left(a, b_{h}\right) \geq \sigma\left(a^{\prime}, b_{h}\right)$ and $\sigma\left(b_{h}, a\right) \leq \sigma\left(b_{h}, a^{\prime}\right), h=1, \ldots, q$. When applying the descending rule, if $t$ is the first value of $h$ such that $\sigma\left(a, b_{t}\right) \geq \lambda$ and if $s$ is the first value of $t$ such that $\sigma\left(a^{\prime}, b_{s}\right) \geq \lambda$, then one necessarily has $t \geq s$. If $t>s$ and $C_{s}$ is selected for the assignment of $a^{\prime}$, then a better category, $C_{t}$ or $C_{t+1}$, is selected for the assignment of $a$. If $t>s$ and $C_{s+1}$ is selected for the assignment of $a^{\prime}$, then the same category, $C_{s+1}$, or a better category is selected for the assignment of $a$. Since $\rho(a, b)$ fulfills Property 2 , if $t=s$, then the monotonicity is also fulfilled.

b.3.1) Stability under a merging operation:

Assume that the consecutive categories $C_{h}$ and $C_{h+1}$ are merged to become a new one, denoted $C_{h}^{\prime}$. Let $b_{h}^{\prime}$ denote the characteristic action introduced to define the new category $C_{h}^{\prime}$. After this modification, the two adjacent categories of $C_{h}^{\prime}$ are $C_{h-1}$ and $C_{h+2}$. From the conditions imposed to $b_{h}^{\prime}$ according to the merging operation (see Definition 3.a)), the new set of characteristic actions $B^{\prime}$ obtained from $B$ when replacing $b_{h}$ and $b_{h+1}$ by $b_{h}^{\prime}$ one has $\sigma\left(b_{h}^{\prime}, b_{h+2}\right)<1$ and $\sigma\left(b_{h-1}, b_{h}^{\prime}\right)<1$. According to the descending rule, we will prove successively that:

1) Any action $a$ previously assigned to a non-adjacent category $C_{s}, s \geq(t+3)$, then $a$ will be assigned to the same category, after modification.

2) Any action $a$ previously assigned to a non-adjacent category $C_{s}, s \leq(t-2)$, then $a$ will be assigned to the same category, after modification.

3) Any action $a$ previously assigned to the adjacent category $C_{t+2}$, then $a$ will either be assigned to the same category or to the new category, $C_{t}^{\prime}$, after modification.

4) Any action $a$ previously assigned to the adjacent category $C_{t-1}$, then $a$ will either be assigned to the same category or to the new category, $C_{t}^{\prime}$, after modification.

5) Any action $a$ previously assigned to the merged category, $C_{t}$ or $C_{t+1}$, then $a$ will either be assigned to the new category $C_{t}^{\prime}$ or to an adjacent category, $C_{t-1}$ or $C_{t+2}$, after modification.

Let us prove these five cases:

1) This proof is trivial since there are no changes in the characteristic actions $b_{t+2}$ and $b_{t+3}$, which are relevant to an assignment to category $C_{t+3}$, after modification. Similar analysis is applied to categories $C_{s}, s \geq(t+4)$.

2) This proof is trivial since there are no changes in the characteristic actions $b_{t-2}$ and $b_{t-1}$, which are relevant to an assignment to category $C_{t-2}$, after modification. Similar analysis is applied to categories $C_{s}, s \leq(t-3)$.

3) An action $a$ was assigned to $C_{t+2}$ if and only if one of the two following conditions holds: 
i) $\sigma\left(a, b_{t+2}\right) \geq \lambda$ and $\sigma\left(a, b_{t+3}\right)<\lambda$, with $\rho\left(a, b_{t+2}\right)>\rho\left(a, b_{t+3}\right)$. In such a case, the proof is similar to 1$)$.

ii) $\sigma\left(a, b_{t+2}\right)<\lambda$ and $\sigma\left(a, b_{t+1}\right) \geq \lambda$, with $\rho\left(a, b_{t+1}\right) \leq \rho\left(a, b_{t+2}\right)$. After a merging operation as analyzed above, the comparison between action $a$ and $b_{t+2}$ does not change, i.e., $\sigma\left(a, b_{t+2}\right)<\lambda$. When comparing action $a$ with the new characteristic action $b_{t}^{\prime}$, one necessarily obtains $\sigma\left(a, b_{t}^{\prime}\right) \geq \lambda$ since $\sigma\left(a, b_{t+1}\right) \geq \lambda$ and $\sigma\left(a, b_{t+1}\right) \leq \sigma\left(a, b_{t}^{\prime}\right)$. Therefore, if $\rho\left(a, b_{t}^{\prime}\right)>\rho\left(a, b_{t+2}\right)$, then action $a$ is assigned to $C_{t}^{\prime}$; otherwise, $a$ is assigned to $C_{t+2}$.

4) An action $a$ was assigned to $C_{t-1}$ if and only if one of the two following conditions holds:

i) $\sigma\left(a, b_{t-1}\right)<\lambda$ and $\sigma\left(a, b_{t-2}\right) \geq \lambda$, with $\rho\left(a, b_{t-2}\right) \leq \rho\left(a, b_{t-1}\right)$. In such a case, the proof is similar to 2$)$.

ii) $\sigma\left(a, b_{t-1}\right) \geq \lambda$ and $\sigma\left(a, b_{t}\right)<\lambda$, with $\rho\left(a, b_{t-1}\right)>\rho\left(a, b_{t}\right)$. After a merging operation as analyzed above, the comparison between action $a$ and $b_{t-1}$ does not change, i.e., $\sigma\left(a, b_{t-1}\right) \geq \lambda$. When comparing action $a$ with the new characteristic action $b_{t}^{\prime}$, one necessarily obtains $\sigma\left(a, b_{t}^{\prime}\right)<\lambda$ since $\sigma\left(a, b_{t}\right)<\lambda$ and $\sigma\left(a, b_{t}^{\prime}\right) \leq \sigma\left(a, b_{t}\right)$. Therefore, if $\rho\left(a, b_{t-1}\right)>\rho\left(a, b_{t}^{\prime}\right)$, then action $a$ is assigned to $C_{t-1}$; otherwise, $a$ is assigned to $C_{t}^{\prime}$.

5) Two cases must be analyzed:

Case 1 - An action $a$ was assigned to $C_{t}$ if and only if one of the two following conditions holds:

i) $\sigma\left(a, b_{t}\right)<\lambda$ and $\sigma\left(a, b_{t-1}\right) \geq \lambda$, with $\rho\left(a, b_{t-1}\right) \leq \rho\left(a, b_{t}\right)$. This proof is similar to 4).ii). In such a case, due to a merging operation, action $a$ will be assigned to $C_{t-1}$ or to $C_{t}^{\prime}$, after modification.

ii) $\sigma\left(a, b_{t}\right) \geq \lambda$ and $\sigma\left(a, b_{t+1}\right)<\lambda$, with $\rho\left(a, b_{t}\right)>\rho\left(a, b_{t+1}\right)$. After a merging operation as analyzed above, the characteristic actions $b_{t}$ and $b_{t+1}$ do not exist anymore. The comparison between action $a, b_{t-1}$, and $b_{t+2}$ do not change, i.e., $\sigma\left(a, b_{t-1}\right) \geq \lambda$ and $\sigma\left(a, b_{t+2}\right)<\lambda$. Therefore, whatever the way action $a$ is compared to the new characteristic action $b_{t}^{\prime}$, $a$ will be assigned to $C_{t-1}\left(\right.$ when $\left.\rho\left(a, b_{t-1}\right)>\rho\left(a, b_{t}^{\prime}\right)\right)$, to $C_{t}^{\prime}\left(\right.$ when $\rho\left(a, b_{t}^{\prime}\right)>\rho\left(a, b_{t+2}\right)$ or $\left.\rho\left(a, b_{t-1}\right) \leq \rho\left(a, b_{t}^{\prime}\right)\right)$, or to $C_{t+2}\left(\right.$ when $\left.\rho\left(a, b_{t}^{\prime}\right) \leq \rho\left(a, b_{t+2}\right)\right)$.

Case 2 - An action $a$ was assigned to $C_{t+1}$ if and only if one of the two following conditions holds:

i) $\sigma\left(a, b_{t+1}\right) \geq \lambda$ and $\sigma\left(a, b_{t+2}\right)<\lambda$, with $\rho\left(a, b_{t+1}\right)>\rho\left(a, b_{t+2}\right)$. This proof is similar to 3).ii). In such a case, due to a merging operation, action $a$ will be assigned to $C_{t}^{\prime}$ or to $C_{t+2}$, after modification.

ii) $\sigma\left(a, b_{t+1}\right)<\lambda$ and $\sigma\left(a, b_{t}\right) \geq \lambda$, with $\rho\left(a, b_{t}\right) \leq \rho\left(a, b_{t+1}\right)$. This proof is similar to 5).Case 1.ii). In such a case, due to a merging operation, action $a$ will be assigned to $C_{t-1}, C_{t}^{\prime}$ or to $C_{t+2}$, after modification.

\section{b.3.2) Stability under a splitting operation:}

Assume that the category $C_{h}$ is split into two new consecutive categories, denoted $C_{h}^{\prime}$ and $C_{h}^{\prime \prime}$. Let $b_{h}^{\prime}$ denote the characteristic action introduced to define the worst of the two new categories, $C_{h}^{\prime}$, and $b_{h}^{\prime \prime}$ the characteristic action introduced to define the best of the two new categories, $C_{h}^{\prime \prime}$ (see Definition 3.b)). After this modification, the two adjacent categories of the two consecutive new categories $C_{h}^{\prime}$ and $C_{h}^{\prime \prime}$ are $C_{h-1}$ and $C_{h+1}$. According to the descending rule (Definition 4), it is required to prove successively that:

1) Any action $a$ previously assigned to a non-adjacent category $C_{s}, s \geq(t+2)$, then $a$ will be assigned to the same category, after modification.

2) Any action $a$ previously assigned to a non-adjacent category $C_{s}, s \leq(t-2)$, then $a$ will be assigned to the same category, after modification.

3) Any action $a$ previously assigned to the adjacent category $C_{t+1}$, then $a$ will either be assigned to the same category or to the new category, $C_{t}^{\prime \prime}$, after modification.

4) Any action $a$ previously assigned to the adjacent category $C_{t-1}$, then $a$ will either be assigned to the same category or to the new category, $C_{t}^{\prime}$, after modification.

5) Any action $a$ previously assigned to the split category $C_{t}$, then $a$ will be assigned to one of the two new categories, $C_{t}^{\prime}$ and $C_{t}^{\prime \prime}$, after modification.

This proof is similar to the proof of the stability under a merging operation by stating that $C_{t}^{\prime}$ (merging) $=C_{t}$ (splitting),$C_{t}$ (merging) $=C_{t}^{\prime}$ (splitting), $C_{t+1}$ (merging) $=C_{t}^{\prime \prime}$ (splitting), $b_{t}$ (before merging) $=b_{t}^{\prime}$ (splitting), and $b_{t+1}$ (before merging) $=b_{t}^{\prime \prime}$ (splitting). It should be noticed that the merging operation is the "inverse operation" of the splitting one, and vice-versa. 
From the proof of Theorem 1, Table 7 presents the summary of the theoretical results concerning the merging and the splitting operations.

Table 7: Summary results for the descending rule

\begin{tabular}{lll}
\hline Before & Merging operation & Splitting operation \\
\hline$C_{s}, s \leq(t-2)$ & $C_{s}, s \leq(t-2)$ & $C_{s}, s \leq(t-2)$ \\
\hline$C t-1$ & $C t-1$ & $C t-1$ \\
& $C^{\prime} t$ & $C^{\prime} t$ \\
\hline$C t$ & $C t-1$ & $C^{\prime} t$ \\
& $C^{\prime} t$ & $C^{\prime \prime} t$ \\
& $C t+2$ & \\
\hline$C t+1$ & $C t-1$ & $C^{\prime \prime} t$ \\
& $C^{\prime} t$ & $C t+1$ \\
& $C t+2$ & \\
\hline$C t+2$ & $C^{\prime} t$ & $C t+2$ \\
& $C t+2$ & \\
\hline$C_{s}, s \geq(t+3)$ & $C_{s}, s \geq(t+3)$ & $C_{s}, s \geq(t+3)$ \\
\hline
\end{tabular}




\section{A.3 Proof of Corollary 1 (in Section 4)}

This proof is trivial according to the proof of Theorem 1.a). Let us noticed that if the hyper-strict separability condition is fulfilled, then the conformity property also holds.

\section{A.4 Proof of Theorem 2 (in Section 4)}

a) If there is only $\lambda$-preference relations between an action $a$ and all characteristic actions, then the following case occurs (see Proposition 1.a)): $a P^{\lambda} b_{0}, a P^{\lambda} b_{1}, \ldots, a P^{\lambda} b_{h}, b_{h+1} P^{\lambda} a, \ldots, b_{q+1} P^{\lambda} a$, with $0 \leq h \leq q$. According to the descending rule (Definition 4), the highest index $t$ such that an action $a$ is $\lambda$-preferred to $b_{t}$ is $t=h$. Thus, if $\rho\left(a, b_{t}\right)>\rho\left(a, b_{t+1}\right)$, then category $C_{t}$ is selected for the assignment of action $a$; otherwise, $C_{t+1}$ is selected. According to the ascending rule (Definition 5), the lowest index $k$ such that a characteristic action $b_{k}$ is $\lambda$-preferred to an action $a$ is $k=(h+1)$. Thus, if $\rho\left(a, b_{k}\right)>\rho\left(a, b_{k-1}\right)$, then category $C_{k}$ is selected for the assignment of action $a$; otherwise, $C_{k-1}$ is selected. Consequently, both joint rules can provide either the same category $\left(C_{h}\right.$ or $\left.C_{h+1}\right)$ or the descending rule provides the category $C_{h}$ and the ascending rule the category $C_{h+1}$ or vice-versa.

b) If an action $a$ is $\lambda$-indifferent to at least one reference action, then the following case occurs (see Proposition 1.b)): $a P^{\lambda} b_{0}, a P^{\lambda} b_{1}, \ldots, a P^{\lambda} b_{h}, a I^{\lambda} b_{h+1}, \ldots, a I^{\lambda} b_{s}, b_{s+1} P^{\lambda} a, \ldots, b_{q+1} P^{\lambda} a$, with $0 \leq h \leq(q-1)$ and $(h+1) \leq s \leq q$. According to the descending rule (Definition 4), the highest index $t$ such that an action $a$ is $\lambda$-indifferent to $b_{t}$ is $t=s$. Thus, if $\rho\left(a, b_{t}\right)>\rho\left(a, b_{t+1}\right)$, then category $C_{t}$ is selected for the assignment of action $a$; otherwise, $C_{t+1}$ is selected. According to the ascending rule (Definition 5), the lowest index $k$ such that an action $a$ is $\lambda$-indifferent to $b_{k}$ is $k=(h+1)$. Thus, if $\rho\left(a, b_{k}\right)>\rho\left(a, b_{k-1}\right)$, then category $C_{k}$ is selected for the assignment of action $a$; otherwise, $C_{k-1}$ is selected. Consequently, the descending rule provides always a category at least as good as the one provided by the ascending rule because $h<(h+1) \leq s<(s+1)$.

c) If an action $a$ is $\lambda$-incomparable to at least one reference action, then the following case occurs (see Proposition 1.c)): $a P^{\lambda} b_{0}, a P^{\lambda} b_{1}, \ldots, a P^{\lambda} b_{h}, a R^{\lambda} b_{h+1}, \ldots, a R^{\lambda} b_{s}, b_{s+1} P^{\lambda} a, \ldots, b_{q+1} P^{\lambda} a$, with $0 \leq h \leq(q-1)$ and $(h+1) \leq s \leq q$. According to the descending rule (Definition 4), the lowest index $t$ such that an action $a$ is $\lambda$-incomparable to $b_{y}$ is $t=(h+1)$. Thus, if $\rho\left(a, b_{t-1}\right)>\rho\left(a, b_{t}\right)$, then category $C_{t-1}$ is selected for the assignment of action $a$; otherwise, $C_{t}$ is selected. According to the ascending rule (Definition 5), the highest index $k$ such that an action $a$ is $\lambda$-incomparable to $b_{k}$ is $k=s$. Thus, if $\rho\left(a, b_{k+1}\right)>\rho\left(a, b_{k}\right)$, then category $C_{k+1}$ is selected for the assignment of action $a$; otherwise, $C_{k}$ is selected. Consequently, the descending rule provides always a category at most as good as the one provided by the ascending rule because $h<(h+1) \leq s<(s+1)$.

\section{A.5 Proof of Corollary 2 (in Section 4)}

Let assume that action $a$ is $\lambda$-indifferent to at least one characteristic action, $b_{h}$. In such a case, one has (see Proposition 1.b)): $a P^{\lambda} b_{k-1}, a I^{\lambda} b_{k}, \ldots, a I^{\lambda} b_{t}, b_{t+1} P^{\lambda} a$, with $t \geq k$. When applying the descending rule (Definition 4), the highest category, $C_{t}$, where $a I^{\lambda} b_{t}$, is selected for a possible assignment of action $a$, if $\rho\left(a, b_{t}\right)>$ $\rho\left(a, b_{t+1}\right)$. When applying the ascending rule (Definition 5), the lowest category, $C_{k}$, where $a I^{\lambda} b_{k}$, is selected for a possible assignment of action $a$, if $\rho\left(a, b_{k}\right)>\rho\left(a, b_{k-1}\right)$. Therefore, the credibility level that allows to select $C_{k}$ and $C_{t}$ as possible categories to assign action $a$ is:

$$
\lambda^{a}=\min \left\{\sigma\left(a, b_{t}\right), \sigma\left(b_{t}, a\right), \sigma\left(a, b_{k}\right), \sigma\left(b_{k}, a\right)\right\} .
$$

\section{A.6 Proof of Proposition 2 (in Section 4)}

According to Definition 1 and Proposition 1, when the chosen credibility level, $\lambda$, increases, then the cases of $\lambda$-preference (Proposition 1.a)) will not increase, the cases of $\lambda$-indifference (Proposition 1.b)) will not increase too, and the cases of $\lambda$-incomparability (Proposition 1.c)) will not decrease. Let us also notice that the credibility indices $\sigma\left(a, b_{h}\right)$ are non-increasing functions in the set $B$, and the credibility indices $\sigma\left(b_{h}, a\right)$ are non-decreasing functions in the set $B$. Therefore, 
a) This proof is trivial from the results provided by Theorem 1, taking into account that: if the chosen credibility level, $\lambda$, increases, then when applying the descending rule (Definition 4), the previously selected category $C_{t}$ for a possible assignment of action $a$ becomes one of the categories $C_{s}, s \leq t$, and when applying the ascending rule (Definition 5), the previously selected category $C_{k}$ for a possible assignment of action $a$ becomes one of the categories $C_{s}, s \geq k$.

b) The proof is similar to $a$ ), and, particularly, when there is no $\lambda$-indifference anymore.

\section{B Appendix: Variable thresholds}

\section{Generalization of the Formulas (2.1)-(2.3) to take into account variable thresholds}

Let $g_{j}$ be a criterion such that the difference between the best and the worst possible performances allows the definition of several performance levels. It is often the case when the performance is defined by a physical or a monetary measure. In this case, the same values for the indifference and the preference thresholds can be judged inappropriate. For instance, a variable threshold defined as a percentage of one of the two performances can be considered more appropriate. It can also be the case with a verbal scale including an enough number of levels. The way of defining these verbal levels can produce a clear distinction between two consecutive levels in certain zones of the scale and an unclear distinction in other zones. In practice, it is often necessary to take into account variable thresholds instead of constant ones.

As noticed in Section 2, the indifference and the preference thresholds have been introduced to discriminate situations of indifference, weak preference, and strict preference when comparing two actions $a$ and $a^{\prime}$ according to the criterion $g_{j}$. With the constant thresholds this discrimination only takes into account the value of the advantage $g_{j}(a)-g_{j}\left(a^{\prime}\right)$. In other words, the discrimination is based on the amplitude of the range $\left[g_{j}(a), g_{j}\left(a^{\prime}\right)\right]$ independently of the position that this range occupies along the scale of criterion $g_{j}$. The introduction of variable thresholds allows to take into account the position of this range along the scale. This is possible by defining threshold functions, which are non-negative functions, in the two following ways:

1) The thresholds called direct, denoted $q_{j}(\cdot)$ and $p_{j}(\cdot)$, were conceived to characterize the frontier of the indifference zone and the frontier of the preference zone, respectively, while progressing in the scale of the criterion $g_{j}$ in the direction of the increasing preferences. For instance, with a direct preference threshold, $a$ is strictly preferred to $a^{\prime}$ if and only if:

i) $g_{j}(a)-g_{j}\left(a^{\prime}\right) \geq p_{j}\left(g_{j}\left(a^{\prime}\right)\right)$ if the preferences increase when the performances increase too;

ii) $g_{j}\left(a^{\prime}\right)-g_{j}(a) \geq p_{j}\left(g_{j}(a)\right)$ if the preferences increase when the performances decrease.

2) The thresholds called inverse, denoted $q_{j}^{\prime}(\cdot)$ and $p_{j}^{\prime}(\cdot)$, were conceived to characterize the frontier of the indifference zone and the frontier of the preference zone, respectively, while progressing in the scale of the criterion $g_{j}$ in the direction of the decreasing preferences. For instance, with an inverse preference threshold, $a$ is strictly preferred to $a^{\prime}$ if and only if:

i) $g_{j}(a)-g_{j}\left(a^{\prime}\right) \geq p_{j}^{\prime}\left(g_{j}(a)\right)$ if the preferences increase when the performances increase too;

ii) $g_{j}\left(a^{\prime}\right)-g_{j}(a) \geq p_{j}^{\prime}\left(g_{j}\left(a^{\prime}\right)\right)$ if the preferences increase when the performances decrease.

Let us notice that direct and inverse thresholds are particular functions subject to certain coherence conditions. Furthermore, if the analyst opts to define only direct thresholds it is possible to obtain automatically the inverse thresholds, and vice-versa, because these two types of variable thresholds are functionally linked (for more details see, for example, Roy 1996, § 9.3.2, p. 188).

When taking into account the notation and definitions introduced in this appendix, the Formulas (2.1)-(2.2) are generalized in case of variable thresholds as follows:

1) With direct thresholds $q_{j}$ and $p_{j}$ must be replaced respectively by:

i) $q_{j}\left(g_{j}(a)\right)$ and $p_{j}\left(g_{j}(a)\right)$ if the preferences increase when the performances increase too; 
ii) $q_{j}\left(g_{j}\left(a^{\prime}\right)\right)$ and $p_{j}\left(g_{j}\left(a^{\prime}\right)\right)$ if the preferences increase when the performances decrease.

2) With inverse thresholds $q_{j}$ and $p_{j}$ must be replaced respectively by:

i) $q_{j}^{\prime}\left(g_{j}\left(a^{\prime}\right)\right)$ and $p_{j}^{\prime}\left(g_{j}\left(a^{\prime}\right)\right)$ if the preferences increase when the performances increase too;

ii) $q_{j}^{\prime}\left(g_{j}(a)\right)$ and $p_{j}^{\prime}\left(g_{j}(a)\right)$ if the preferences increase when the performances decrease.

The reasons that led us to introduce variable indifference and preference thresholds (rather than constant ones) are the same that led us to introduce variable veto thresholds, in the case where a veto threshold is associated to criterion $g_{j}$. The analyst can again opt between direct veto thresholds, denoted $v_{j}(\cdot)$, and inverse veto thresholds, denoted $v_{j}^{\prime}(\cdot)$.

The Formula (2.3) is generalized as follows:

1) With direct thresholds, $p_{j}$ and $v_{j}$ must be replaced respectively by:

i) $p_{j}\left(g_{j}(a)\right)$ and $v_{j}\left(g_{j}(a)\right)$ if the preferences increase when the performances increase too;

ii) $p_{j}\left(g_{j}\left(a^{\prime}\right)\right)$ and $v_{j}\left(g_{j}\left(a^{\prime}\right)\right)$ if the preferences increase when the performances decrease.

2) With inverse thresholds, $p_{j}$ and $v_{j}$ must be replaced respectively by:

i) $p_{j}^{\prime}\left(g_{j}\left(a^{\prime}\right)\right)$ and $v_{j}^{\prime}\left(g_{j}\left(a^{\prime}\right)\right)$ if the preferences increase when the performances increase too;

ii) $p_{j}^{\prime}\left(g_{j}(a)\right)$ and $v_{j}^{\prime}\left(g_{j}(a)\right)$ if the preferences increase when the performances decrease.

The above analysis of variable thresholds can also be taken into account when analyzing the minimum credibility level associated to the set of characteristic actions as follows (consider the case of a variable preference thresholds):

i) $g_{j}\left(b_{h+1}\right)-g_{j}\left(b_{h}\right)>p_{j}\left(g_{j}\left(b_{h}\right)\right)$ if the preferences increase when the performances increase too;

ii) $g_{j}\left(b_{h+1}\right)-g_{j}\left(b_{h}\right)>p_{j}^{\prime}\left(g_{j}\left(b_{h+1}\right)\right)$ if the preferences increase when the performances decrease.

Finally, let us notice that when using variable thresholds in the sorting problematique it is not necessary to compute the threshold values for each scale levels of criterion $g_{j}$. Indeed, all the Electre TRI-C formulas (the same is applied to EleCtre TrI-B) can be written by making use of the values that are assigned to the direct and inverse thresholds only for the performances of the characteristic actions, $g_{j}\left(b_{h}\right), h=0, \ldots,(q+1)$. It comes from the fact that in a given formula, concerning the way in which two actions $a$ and $a^{\prime}$ are compared, that makes use of a direct threshold computed for $a$ it is possible to substitute it by an inverse threshold computed for $a^{\prime}$, and vice-versa.

\section{References}

André, S. and B. Roy (2007). Conception et mise en place d'un outil d'évaluation de la performance environnementale : Le cas des raffineries de la société TOTAL. Journal of Decision Systems 16(3), 335-367.

Arondel, C. and P. Girardin (2000). Sorting cropping systems on the basis of their impact on groundwater quality. European Journal of Operational Research 127(3), 467-482.

Belacel, N. (2000). Multicriteria assignment method PROAFTN: Methodology and medical application. European Journal of Operational Research 125(1), 175-183.

Costa, H., A. Soares, and P. Oliveira (2004). Evaluation of dangerous materials transport companies using the ELECTRE TRI method. Gestão \& Produção 11(2), 221-229. In Portuguese.

Devaud, J., G. Groussaud, and E. Jacquet-Lagrèze (1980). UTADIS : Une méthode de construction de fonctions d'utilité additives rendant compte de jugements globaux. European Working Group on Multicriteria Decision Aid, Bochum, Germany.

Diakoulaki, D. and V. Hontou (2003). A multicriteria approach to burden sharing among industrial branches for combating climate change. Clean Technologies and Environmental Policy 5(1), 35-40.

Doumpos, M. and C. Zopounidis (2004). A multicriteria classification approach based on pairwise comparisons. European Journal of Operational Research 158(2), 378-389. 
Fernández, E., J. Navarro, and A. Duarte (2008). Multicriteria sorting using a valued preference closeness relation. European Journal of Operational Research 185(2), 673-686.

Figueira, J., S. Greco, and B. Roy (2009). ELECTRE methods with interaction between criteria: An extension of the concordance index. European Journal of Operational Research 199(2), 496-505.

Georgopoulou, E., Y. Sarafidis, S. Mirasgedis, S. Zaimi, and D. Lalas (2003). A multiple criteria decision-aid approach in defining national priorities for greenhouse gases emissions reduction in the energy sector. European Journal of Operational Research 146(1), 199-215.

Joerin, F., M. Thériault, and A. Musy (2001). Using GIS and outranking multicriteria analysis for land-use suitability assessment. International Journal of Geographical Information Science 15(2), 153-174.

Massaglia, R. and A. Ostanello (1991). N-TOMIC: A support system for multicriteria segmentation problems. In P. Korhonen, A. Lewandowski, and J. Wallenius (Eds.), Multiple Criteria Decision Support: Proceedings, Helsinki, Finland, 1989, Volume 356 of Lectures Notes in Economics and Mathematical Systems, pp. 167-174. Berlin: SpringerVerlag.

Matias, S. (2008). A Multicriteria Decision Aiding Assignment Methodology for Assisted Reproductive Technology. Master Dissertation in Biomedicine, Instituto Superior Técnico, Technical University of Lisbon, Lisbon, Portugal.

Mavrotas, G., D. Diakoulaki, and P. Capros (2003). Combined MCDA IP approach for project selection in the electricity market. Annals of Operations Research 120(1), 159-170.

Merad, M., T. Verdel, B. Roy, and S. Kouniali (2004). Use of multi-criteria decision-aids for risk zoning and management of large area subjected to mining-induced hazards. Tunnelling and Underground Space Technology 19, 165-178.

Moscarola, J. and B. Roy (1977). Procédure automatique d'examen de dossiers fondée sur une segmentation trichotomique en présence de critères multiples. R.A.I.R.O. Recherche Opérationnelle / Operations Research 11(2), $145-173$.

Mousseau, V., B. Roy, and I. Sommerlatt (2001). Development of a decision aiding tool for the evolution of public transport ticket pricing in the Paris region. In A. Colorni, M. Paruccini, and B. Roy (Eds.), A-MCD-A: Aide Multicritère à la Décision / Multiple Criteria Decision Aiding, pp. 213-230. Luxembourg: Joint Research Center, European Commision.

Norese, M. and S. Viale (2002). A multi-profile sorting procedure in the public administration. European Journal of Operational Research 138(2), 365-379.

Perny, P. (1998). Multicriteria filtering methods based on concordance and non-discordance principles. Annals of Operations Research 80, 137-165.

Raju, K., L. Duckstein, and C. Arondel (2000). Multicriterion analysis for sustainable water resources planning: A case study in Spain. Water Resources Management 14(6), 435-456.

Rogers, M., M. Bruen, and L. Maystre (2000). ELECTRE and Decision Support: Methods and Aplications in Engineering and Infrastructure Investment. London: Kluwer Academic Publishers.

Roget, F. and X. González (2005). Assigning of public subvention using ELECTRE TRI: An application on the rural tourism in Galicia. Estudios de Economía Aplicada 23(1), 1-24. In Spanish.

Roy, B. (1991). The outranking approach and the foundations of ELECTRE methods. Theory and Decision 31(1), 49-73.

Roy, B. (1993). Decision science or decision-aid science? European Journal of Operational Research 66 (2), $184-203$.

Roy, B. (1996). Multicriteria Methodology for Decision Aiding. Dordrecht: Kluwer Academic Publishers.

Roy, B. (2002). Présentation et interprétation de la méthode ELECTRE TRI pour affecter des zones dans des catégories de risque. Document du LAMSADE 124, Université Paris-Dauphine, Paris, France.

Roy, B. and D. Bouyssou (1993). Aide Multicritère à la Décision : Méthodes et Cas. Production et Techniques Quantitatives Appliquées à la Gestion. Paris, France: Economica.

Roy, B. and R. Słowiński (2008). Handling effects of reinforced preference and counter-veto in credibility of outranking. European Journal of Operational Research 188(1), 185-190.

Roy, B. and P. Vincke (1984). Relational systems of preference with one or more pseudo-criteria: Some new concepts and results. Management Science 30(11), 1323-1335.

Siskos, Y., E. Grigoroudis, E. Krassadaki, and N. Matsatsinis (2007). A multicriteria accreditation system for information technology skills and qualifications. European Journal of Operational Research 182(2), 867-885.

Słowiński, R. and J. Stefanowski (1994). Rough classification with valued closeness relation. In E. Diday, Y. Lechevallier, M. Schader, P. Bertrand, and B. Burtschy (Eds.), New Approaches in Classification and Data Analysis, pp. $482-489$. Berlin, Germany: Springer-Verlag.

Tervonen, T., J. Figueira, R. Lahdelma, J. Almeida Dias, and P. Salminen (2009). A stochastic method for robustness analysis in sorting problems. European Journal of Operational Research 192(1), 236-242.

Yu, W. (1992). Aide Multicritère à la Décision dans le Cadre de la Problématique du Tri : Concepts, Méthodes et Applications. Thèse de Doctorat, LAMSADE, Université Paris-Dauphine, Paris, France. 
Zopounidis, C. and M. Doumpos (2002). Multi-criteria decision aid in financial decision making: Methodologies and literature review. Journal of Multi-Criteria Decision Analysis 11(4-5), 167-186. 